\title{
Analyses of high mass resonances at ATLAS and CMS
}

\author{
L.R. Flores-Castillo \\ University of Wisconsin-Madison
}

On behalf of the ATLAS and CMS collaborations 


\section{Outline}

- Introduction

- Detectors

- Dileptons: ee, $\mu \mu, \tau \tau$

- Lepton-neutrino

- Lepton(s) plus jet(s)

- Dijets

- Conclusion and outlook 


\section{Introduction}

- Although extremely successful, there are indications that the Standard Model (SM) is not a complete theory

- Plausible SM extensions predict narrow states that can be reconstructed (completely or not) in ATLAS and CMS

- Dileptons $(e, \mu, \tau)$ :

- New heavy gauge bosons ( $\left.Z^{\prime}\right)$, KK resonances, gravitons

- Diphotons (see H. Hadavand's talk)

- Lepton + Missing Transverse Energy

- New heavy W-like boson

- Leptons plus Jets

- Leptoquarks

- Left-Right Symmetric Models

- Dijets (see K. Terashi's talk)

- Results shown correspond to a center of mass energy of $14 \mathrm{TeV}$

- At $10 \mathrm{TeV}$, cross sections 50\%-75\% smaller in 100GeV to $1 \mathrm{TeV}$ 


\section{LHC}

- Operating parameters

- $\mathrm{E}_{\text {beam }}=7 \mathrm{TeV} \rightarrow 5 \mathrm{TeV}$

- $\mathrm{L}=10^{34} \mathrm{~cm}^{-2} \mathrm{~s}^{-1} \rightarrow 10^{30}-10^{32} \mathrm{~cm}^{-2} \mathrm{~s}^{-1}$

- Bunch Spacing = 25 ns (40 MHz)

- Pile-Up $=2-20$ collisions/crossing

- Duration of collisions $\approx 10-24 \mathrm{~h}$

- Down Time $\approx 1.5 \mathrm{~h}$

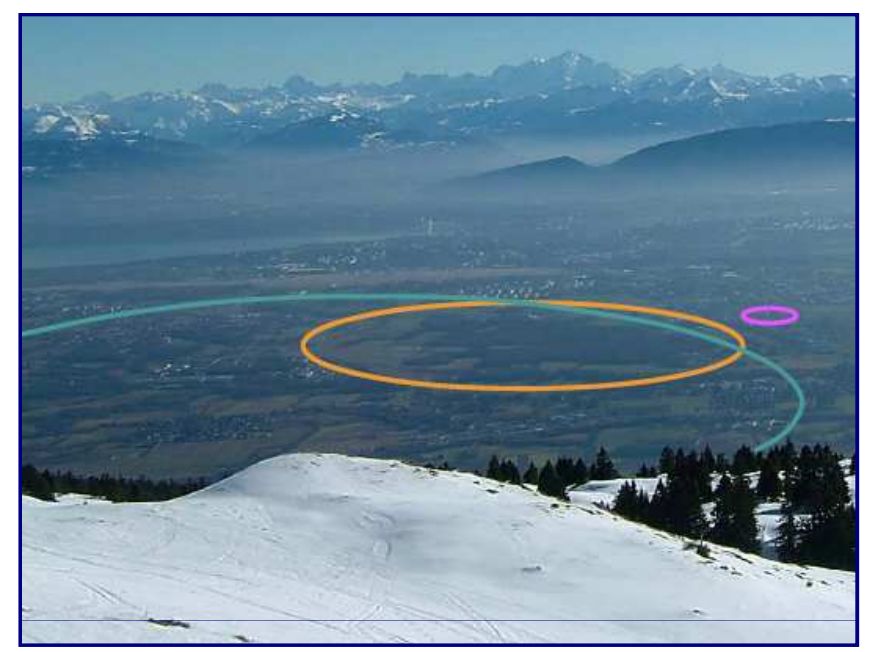

\begin{tabular}{|l|c|c|c|c|}
\hline Example Decay Channel & $\begin{array}{c}\text { LEP } \\
\text { (all) }\end{array}$ & $\begin{array}{c}\text { Tevatron } \\
\text { (all) }\end{array}$ & $\begin{array}{c}\text { LHC } \\
100 \mathrm{pb}^{-1}\end{array}$ & $\begin{array}{c}\text { LHC } \\
1 \mathrm{fb}^{-1}\end{array}$ \\
\hline $\mathrm{W} \rightarrow \mu \mathrm{V}$ & $\sim 10^{4}$ & $\sim 10^{6}$ & $\sim 10^{6}$ & $\sim 10^{7}$ \\
\hline $\mathrm{Z} \rightarrow \mu \mu$ & $\sim 10^{6}$ & $\sim 10^{5}$ & $\sim 10^{5}$ & $\sim 10^{6}$ \\
\hline $\mathrm{tt} \rightarrow \mathrm{WbWb} \rightarrow \mu \mathrm{V}+\mathrm{X}$ & & $\sim 10^{4}$ & $\sim 10^{4}$ & $\sim 10^{5}$ \\
\hline $\mathrm{QCD}$ jets $\left(\mathrm{p}_{\mathrm{T}}>1 \mathrm{TeV}\right)$ & & & $\sim 10^{3}$ & $\sim 10^{4}$ \\
\hline $\mathrm{Z}^{\prime}(1 \mathrm{TeV}) \rightarrow \mu \mu$ & & & $\sim 20$ & $\sim 10^{2}$
\end{tabular}




\section{Detectors}
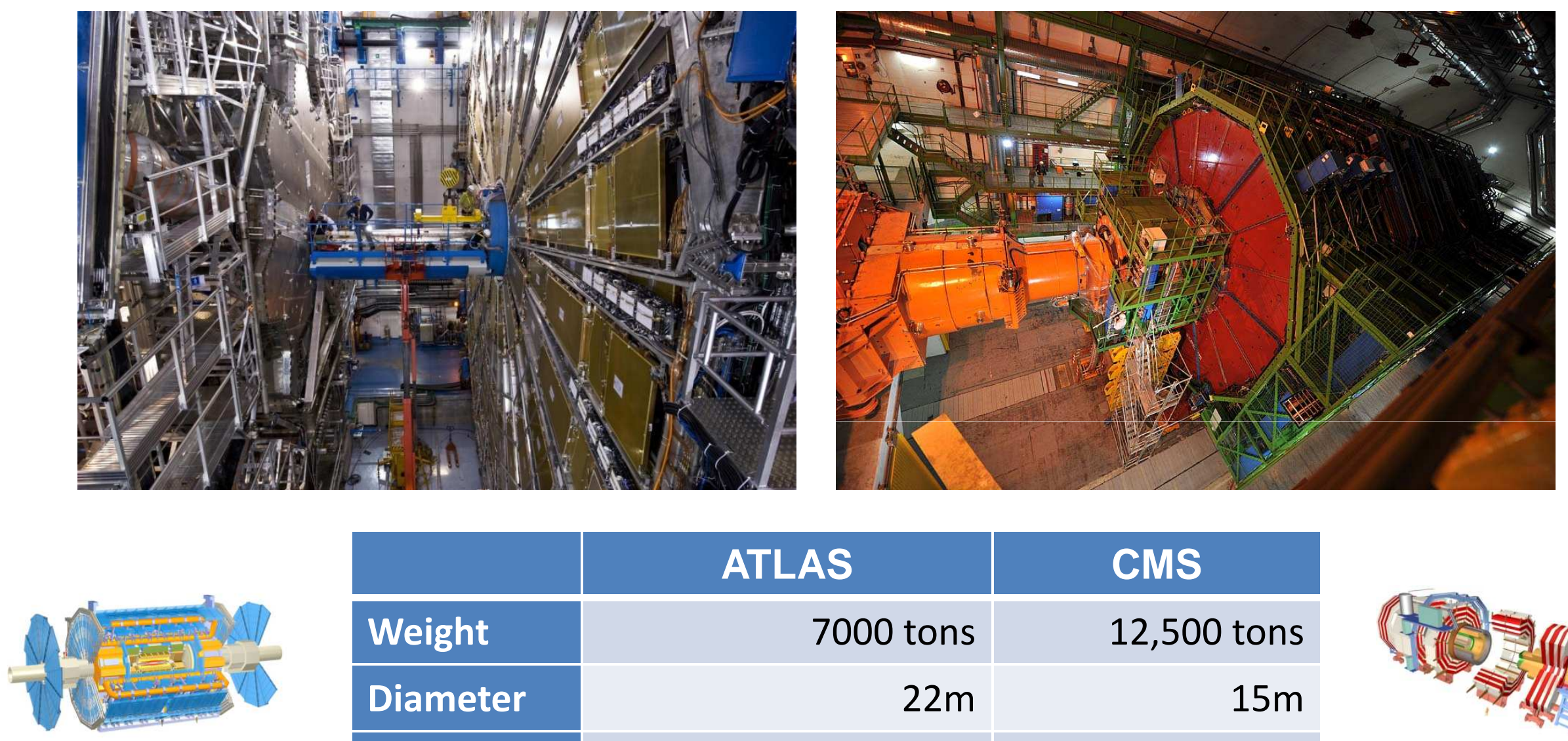

\begin{tabular}{l|r|r|} 
& ATLAS & \multicolumn{2}{|c|}{ CMS } \\
\hline Weight & 7000 tons & 12,500 tons \\
\hline Diameter & $22 \mathrm{~m}$ & $15 \mathrm{~m}$ \\
\hline Length & $46 \mathrm{~m}$ & $22 \mathrm{~m}$ \\
\hline $\begin{array}{l}\text { Peak } \\
\text { B Field }\end{array}$ & 2T solenoid & 4T solenoid \\
& 3.9T (peak) BA toroid & \\
& 4.1T (peak) EC toroids & \\
\end{tabular}

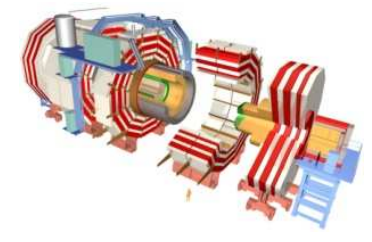




\section{Bending power}

Rather than the peak values of the magnetic field, tracking capabilities depend on the bending power of the field configuration

$$
\int \mathrm{d} \alpha=\int \frac{\mathrm{d} s}{R}=\int\left|\frac{\mathrm{d}^{2} \boldsymbol{r}}{\mathrm{d} s^{2}}\right| \mathrm{d} s=\frac{q}{p} \int\left|\frac{\mathrm{d} \boldsymbol{r}}{\mathrm{d} s} \times \boldsymbol{B}(\boldsymbol{r})\right| \mathrm{d} s \quad \begin{aligned}
& R: \text { radius of curvature } \\
& s: \text { distance along the trajectory } \\
& \boldsymbol{r} \text { : position vector }
\end{aligned}
$$

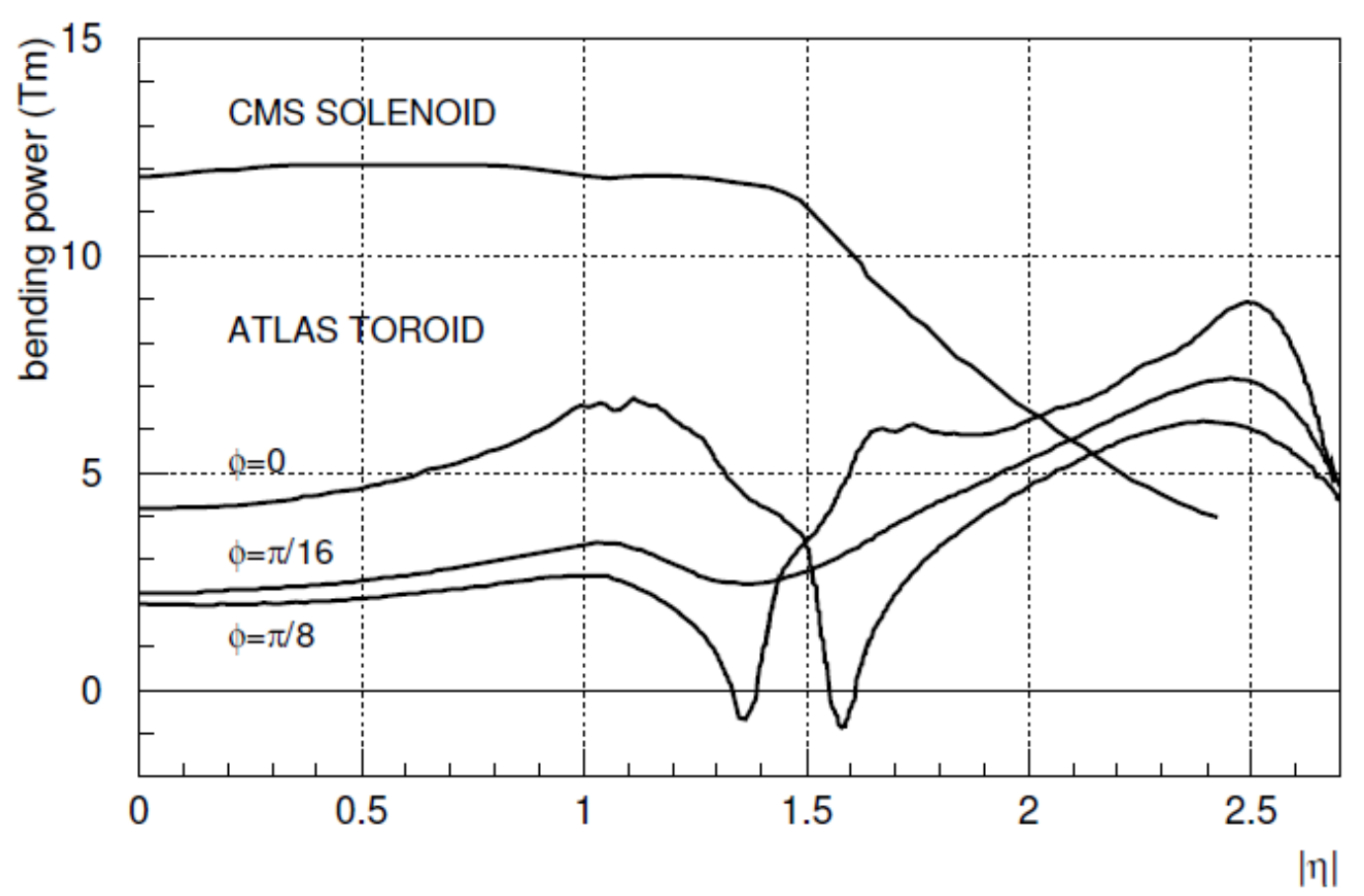




\section{Performance}

\begin{tabular}{|c|c|c|}
\hline & ATLAS & CMS \\
\hline Tracker & $\begin{array}{l}\text { Si pixels, strips }+ \text { TRT (pid) } \\
\sigma / p_{T} \approx 5 \times 10^{-4} p_{T} \oplus 0.01\end{array}$ & $\begin{array}{l}\text { Si pixels, strips } \\
\sigma / p_{T} \approx 1.5 \times 10^{-4} p_{T} \oplus 0.005\end{array}$ \\
\hline EM calorimeter & $\begin{array}{l}\mathrm{Pb}+\mathrm{LAr} \\
\sigma / \mathrm{E} \approx 10 \% / \sqrt{ } \mathrm{E} \oplus 0.007\end{array}$ & $\begin{array}{l}\mathrm{PbWO}_{4} \text { crystals } \\
\sigma / \mathrm{E} \approx 2-5 \% / \sqrt{ } \mathrm{E} \oplus 0.005\end{array}$ \\
\hline Hadronic calorimeter & $\begin{array}{l}\text { Fe+scintillator } / \mathrm{Cu}+\mathrm{Lar} \\
\sigma / \mathrm{E} \approx 50 \% / \sqrt{\mathrm{E}} \oplus 0.03\end{array}$ & $\begin{array}{l}\text { Cu+scintillator } \\
\sigma / E \approx 100 \% / \sqrt{ } E \oplus 0.05\end{array}$ \\
\hline Combined Muons (ID+MS) & 2\%@50GeV to 10\%@1TeV & 1\%@50GeV to 5\%@1TeV \\
\hline
\end{tabular}

- Powerful id:

- Photons: Jet rejection $\sim$ few $10^{3}$ for $\sim 80 \%$ photon efficiency

- Electrons: Jet rejection $\sim 10^{5}$ for $\sim 60 \%$ electron efficiency

- B-jets: $\quad$ Light flavor jet rejection $\sim 100$ for $\sim 60 \%$ efficiency

- $\tau \rightarrow$ hadrons: Jet rejection $\sim$ few hundreds for $\sim 50 \%$ efficiency

- Missing transverse momentum and jet reconstruction 


\section{DILEPTONS}

e, $\mu$ 


\section{Signature, selection}

- Relatively clean signatures

- Good mass resolution

- Easy to trigger on

- Backgrounds

- Main background: SM Drell-Yan

- tt, dijets, W+jets, gamma+jets

- Selection

- 2 well reconstructed, isolated leptons

- $\mid$ eta $\mid<2.5$ (except muons in CMS, 2.4)

- pT>30 or $50 \mathrm{GeV}$

- Opening angle: not needed for discovery, but useful to help distinguish models

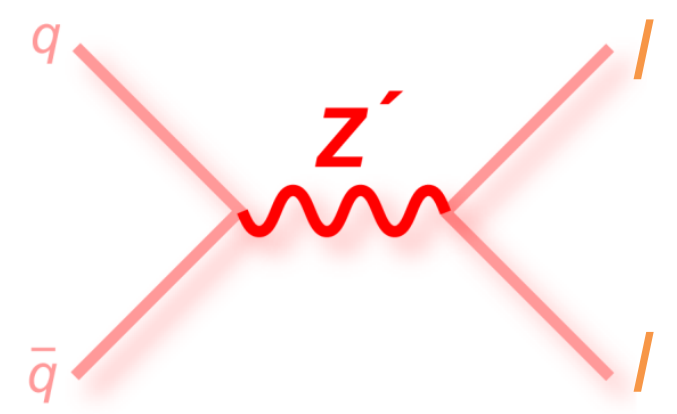

CDF Run II Preliminary

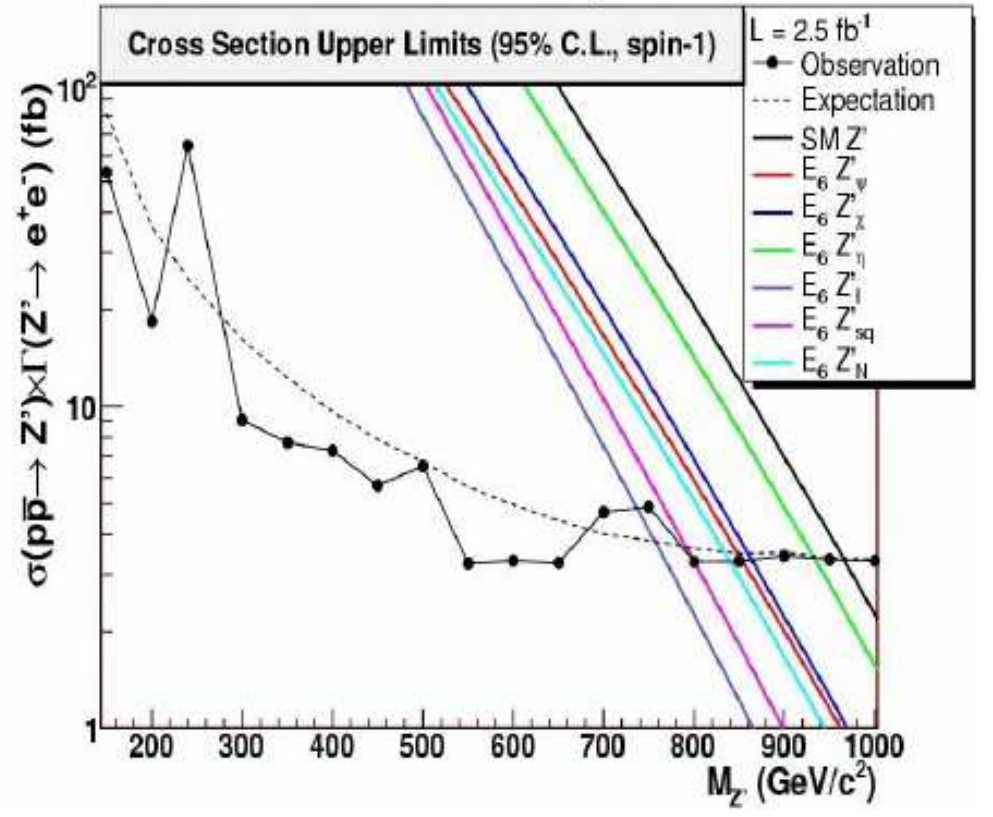




\section{Backgrounds}

- Drell-Yan

- Processes where jets or photons fake electrons

- Electron and muons from Z and W

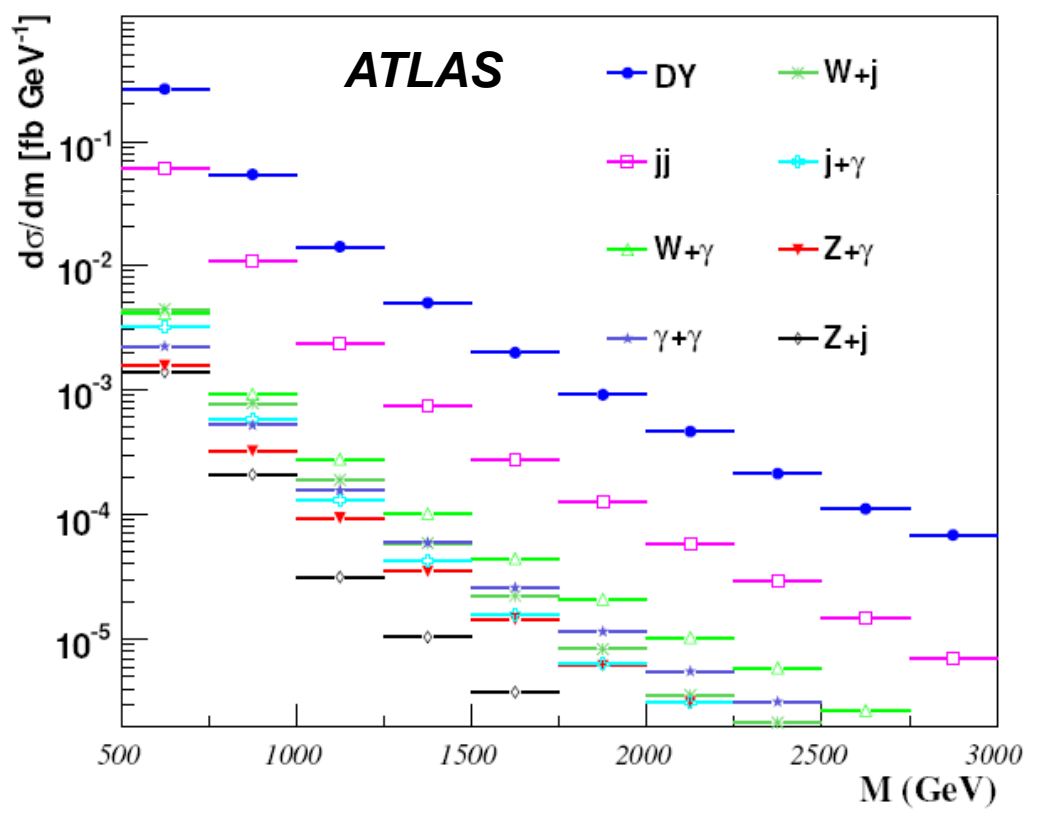

Generator-level estimation after applying current values of jet and photon rejection (10 ${ }^{4}$ and 10 , respectively)

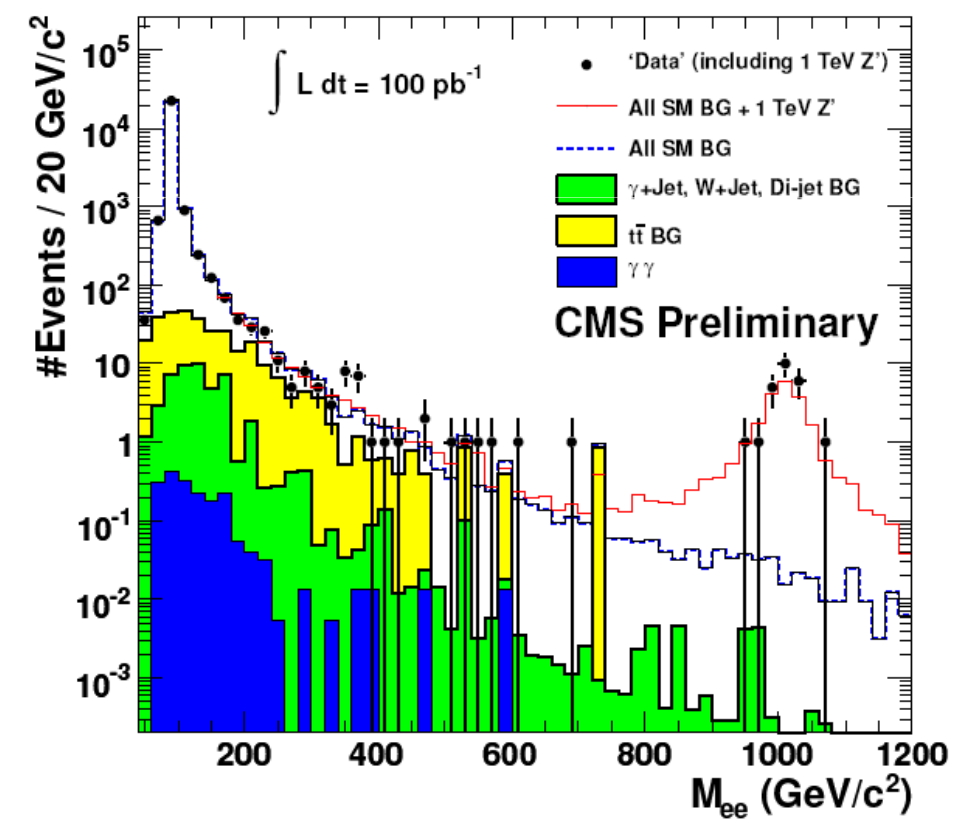

Full detector simulation 


\section{Background estimation}

- Detector effects and theoretical uncertainties can affect the background estimation

- Control sample strategies can help constrain some of these backgrounds
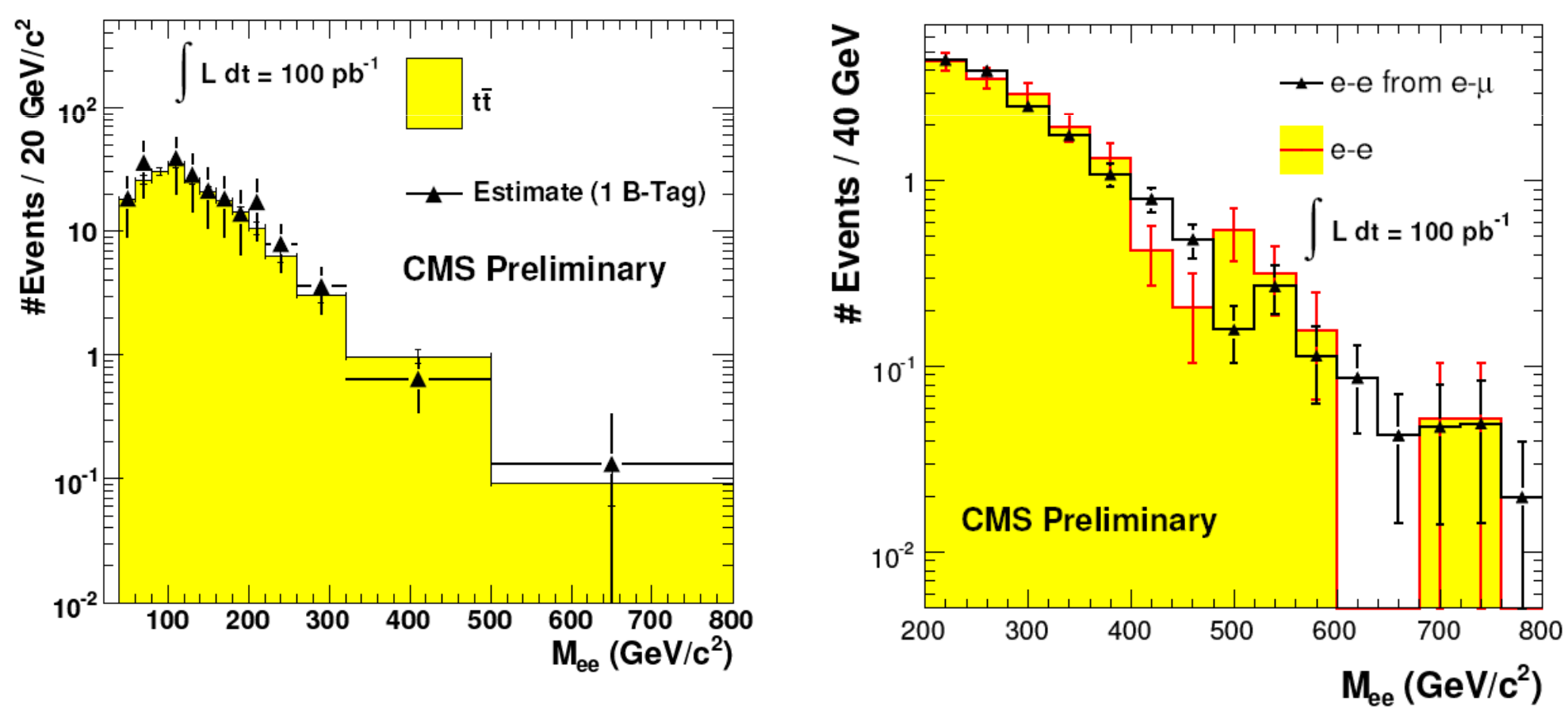


\section{Electrons at high pT}

- Reconstruction and identification optimized for high energy electrons

- Robust criteria based on shower shape, track matching, isolation

- Efficiency 80\%

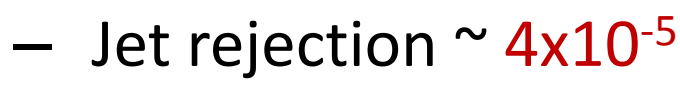

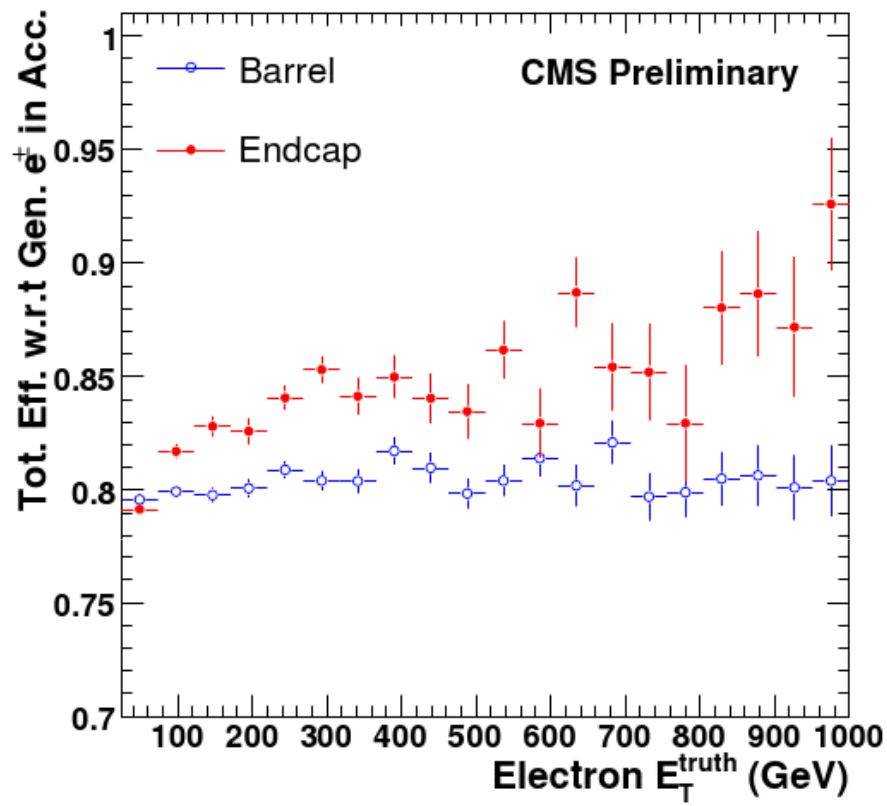

- ECAL saturation (CMS)

- Large energy deposit in one crystal (1.7, $3 \mathrm{TeV}$ for barrel, endcap)

- Can be recovered using surrounding crystals 


\section{Muon system alignment}
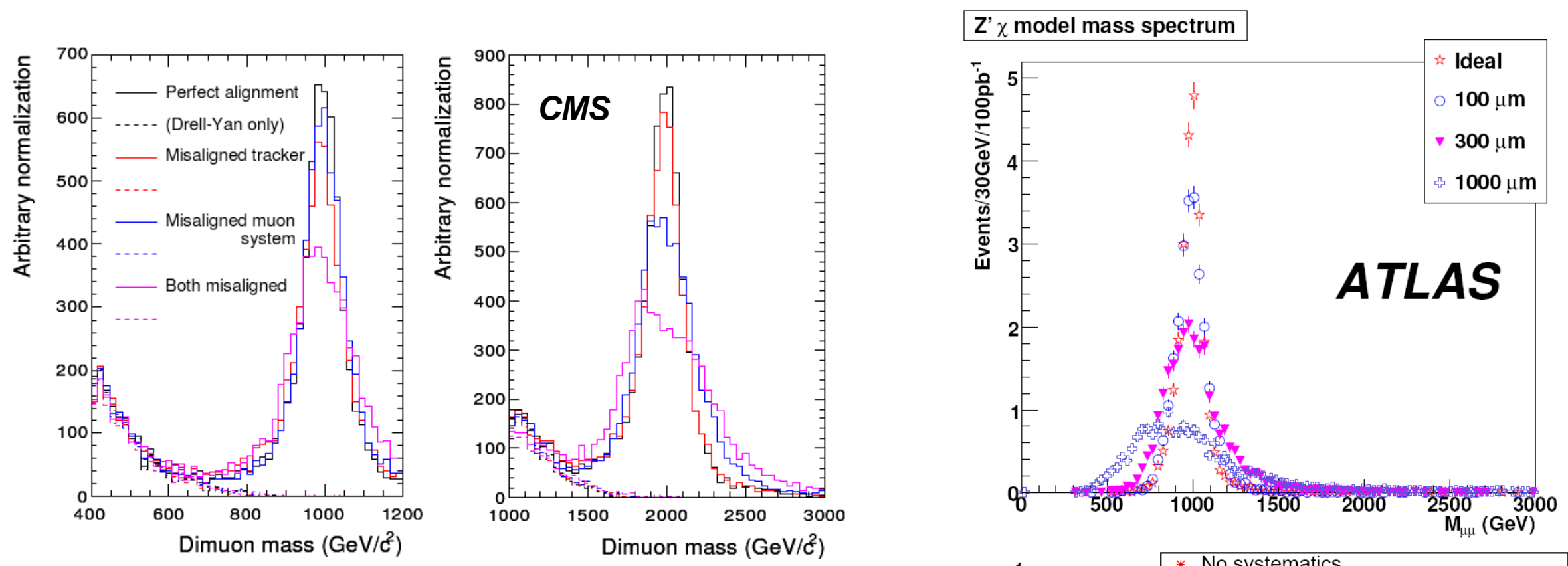

- Both experiments have evaluated the effect of possible scenarios

- Strong effect on physics potential

- Roughly twice as much luminosity needed for discovery

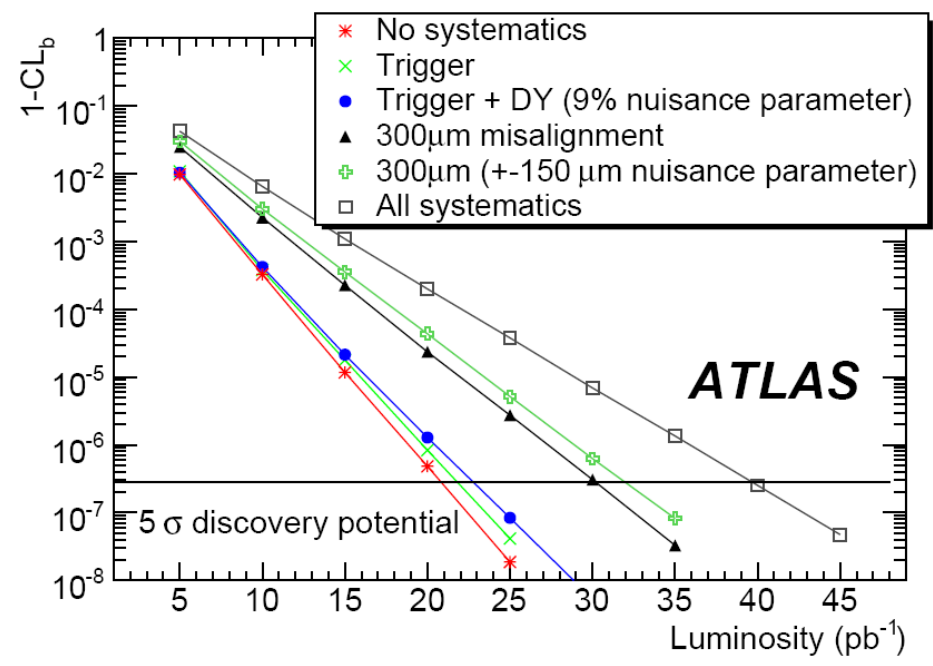




\section{Discovery potential}

- Factorization of the PDF

Four parameters $\left(\Gamma_{Z}, A_{\text {peak }}, A_{\text {interf }}, M_{Z^{\prime}}\right)$

$$
\begin{aligned}
\left.\frac{d \sigma}{d m}\right|_{\text {Signal }}(m) & =\frac{1}{m^{2}} \times G_{P D F}(m) \\
& +\mathscr{A}_{\text {peak }} \times \frac{\Gamma_{Z^{\prime}}^{2}}{m_{Z^{\prime}}^{2}} \frac{m^{2}}{\left(m^{2}-m_{Z^{\prime}}^{2}\right)^{2}+m_{Z^{\prime}}^{2} \Gamma_{Z^{\prime}}^{2}} \times G_{P D F}(m) \\
& +\mathscr{A}_{\text {interf }} \times \frac{\Gamma_{Z^{\prime}}^{2}}{m_{Z^{\prime}}^{2}} \frac{m^{2}-m_{Z^{\prime}}^{2}}{\left(m^{2}-m_{Z^{\prime}}^{2}\right)^{2}+m_{Z^{\prime}}^{2} \Gamma_{Z^{\prime}}^{2}} \times G_{P D F}(m)
\end{aligned}
$$

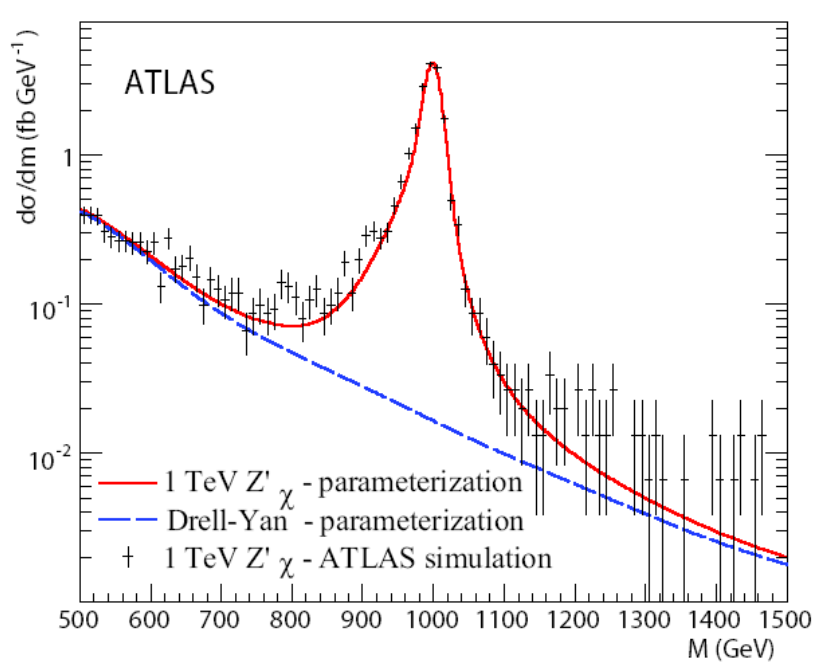

$\otimes$ Resolution (from full simulation)

$\times$ acceptance (depends on the model)

$\times$ efficiency of cuts

- Statistical analysis:

- Log-likelihood ratio estimator (LLR)

- Signal+Background and Background-Only LLR distributions used to compute $\mathrm{CL}_{\mathrm{s}}$

- No need for an optimized mass window

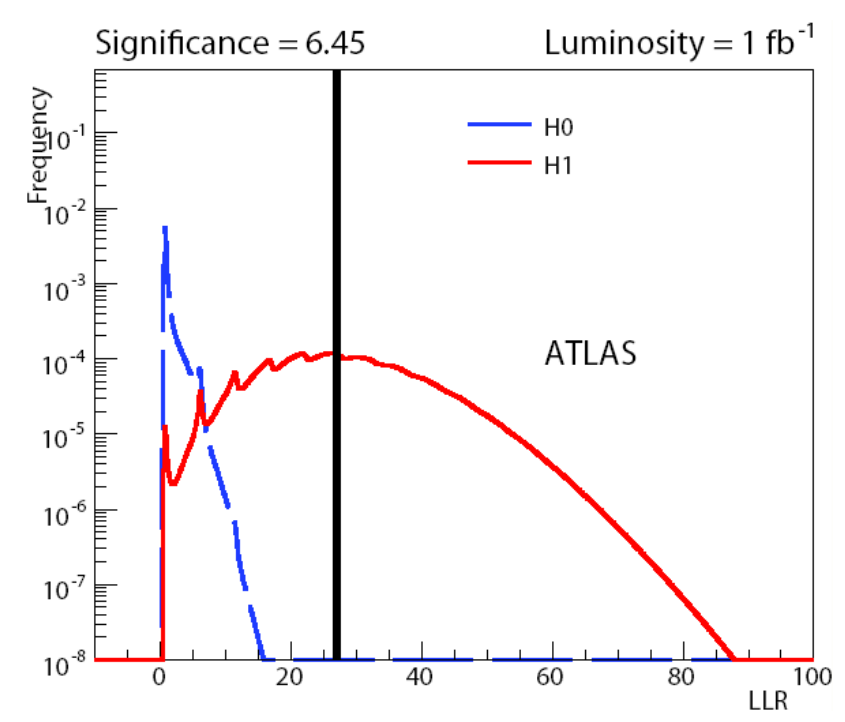




\section{"Look-elsewhere"}

- "Trials factor", "greedy bump bias", ...

- Background fluctuations anywhere in the full mass range under study increase the probability of a fake discovery

- Studied with toy MC using a (max Likelihood) fit-based approach; floating vs fixed mass fits

- Degradation of the significance of $\sim 15 \%$
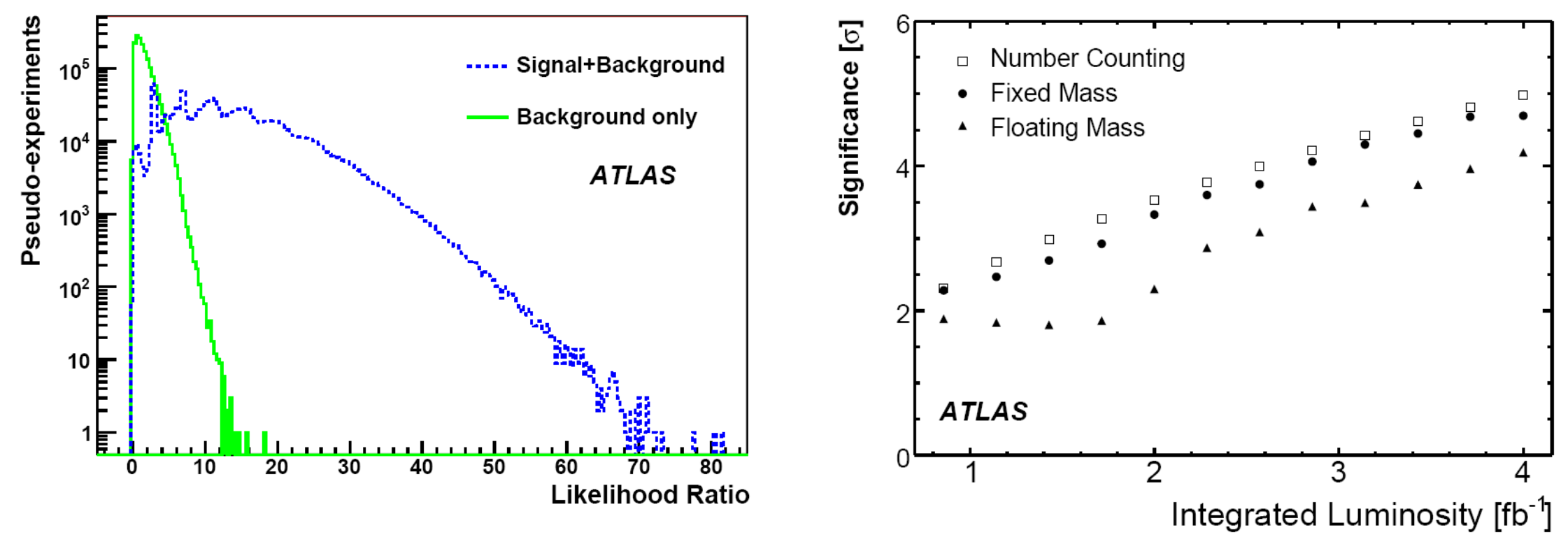


\section{Sensitivity, reach}

- Comparable reach for both experiments

- If slightly above the current Tevatron limit (1TeV), as low as $100 \mathrm{pb}^{-1}$ of physics data could yield a $5 \sigma$ discovery
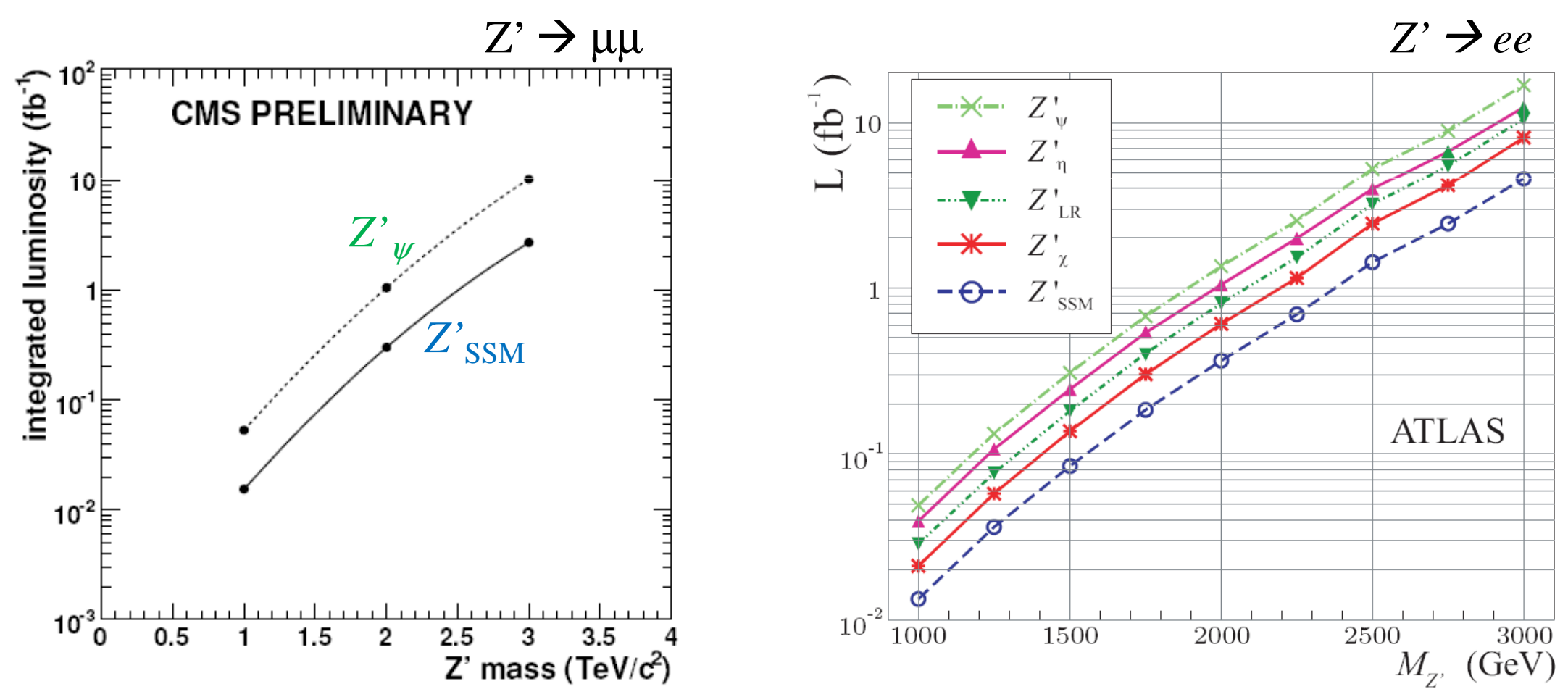


\section{From 14 to $10 \mathrm{TeV}$}
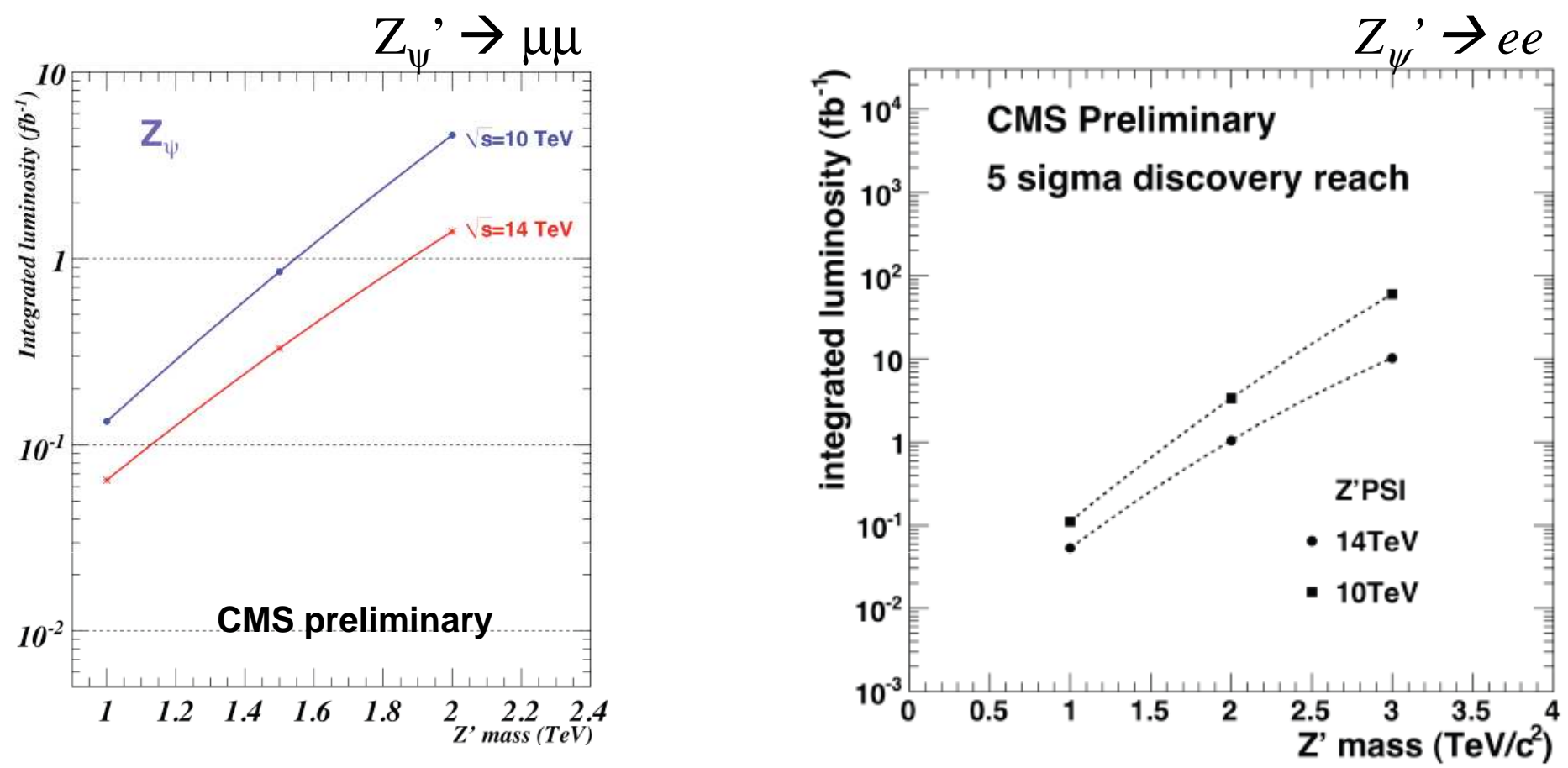

- Both experiments have assessed the effect of the lowered centerof-mass energy

- Production cross sections are reduced by factors $\sim 2$ or 3 (for masses of the $Z^{\prime}$ between 1 and $2 \mathrm{TeV}$ )

- Accordingly, the luminosity needed for a $5 \sigma$ discovery $\sim$ doubles 


\section{Gravitons}

- Randall-Sundrum models
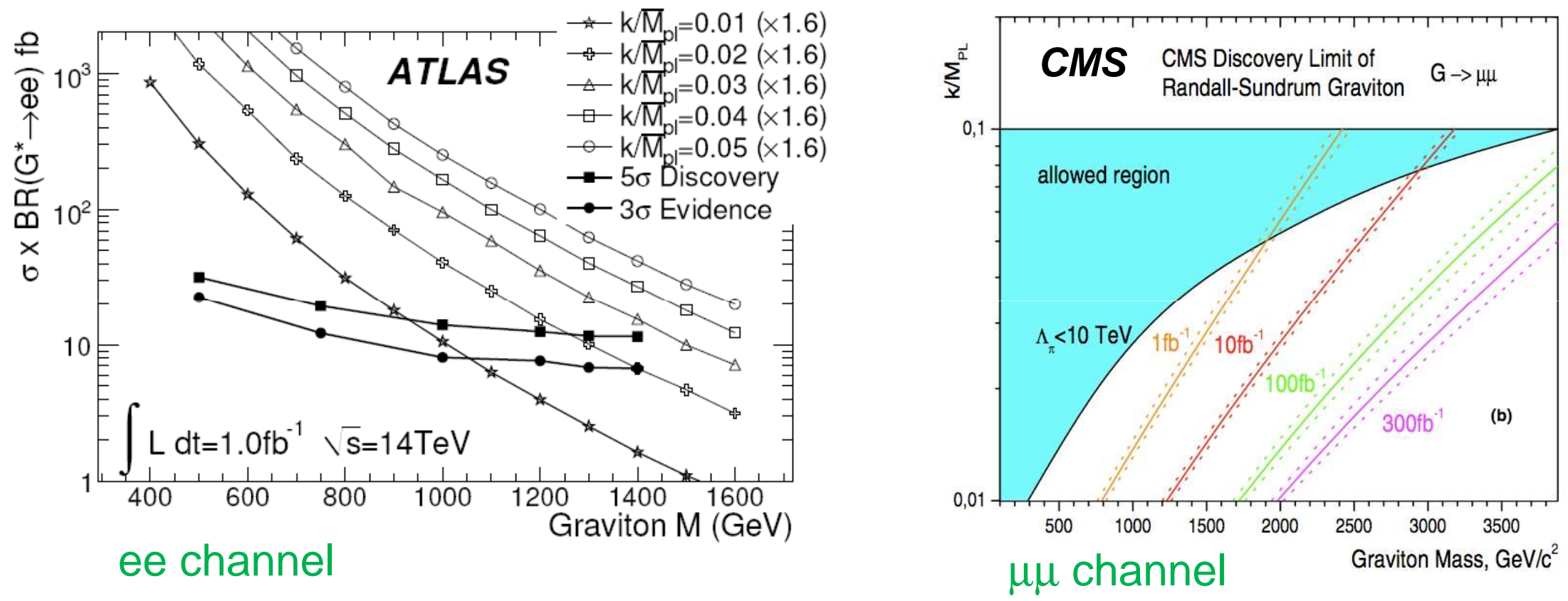

- Treatment: mass floating in the full mass range, width fixed to detector resolution

- For some values of $\mathrm{k} / \mathrm{M}_{\mathrm{pl}}$, possible discovery with $\mathrm{O}\left(100 \mathrm{pb}^{-1}\right)$ 


\section{Technicolor}

- Lowest mass states: $\pi_{\mathrm{T}}, \rho_{\mathrm{T}}, \omega_{\mathrm{T}}$

- $\rho_{\mathrm{T}}, \omega_{\mathrm{T}}$ can decay into fermion-antifermion pairs

- "Technicolor Strawman Model"; $\rho_{T}, \omega_{T}$ nearly degenerate

- Dimuon model:
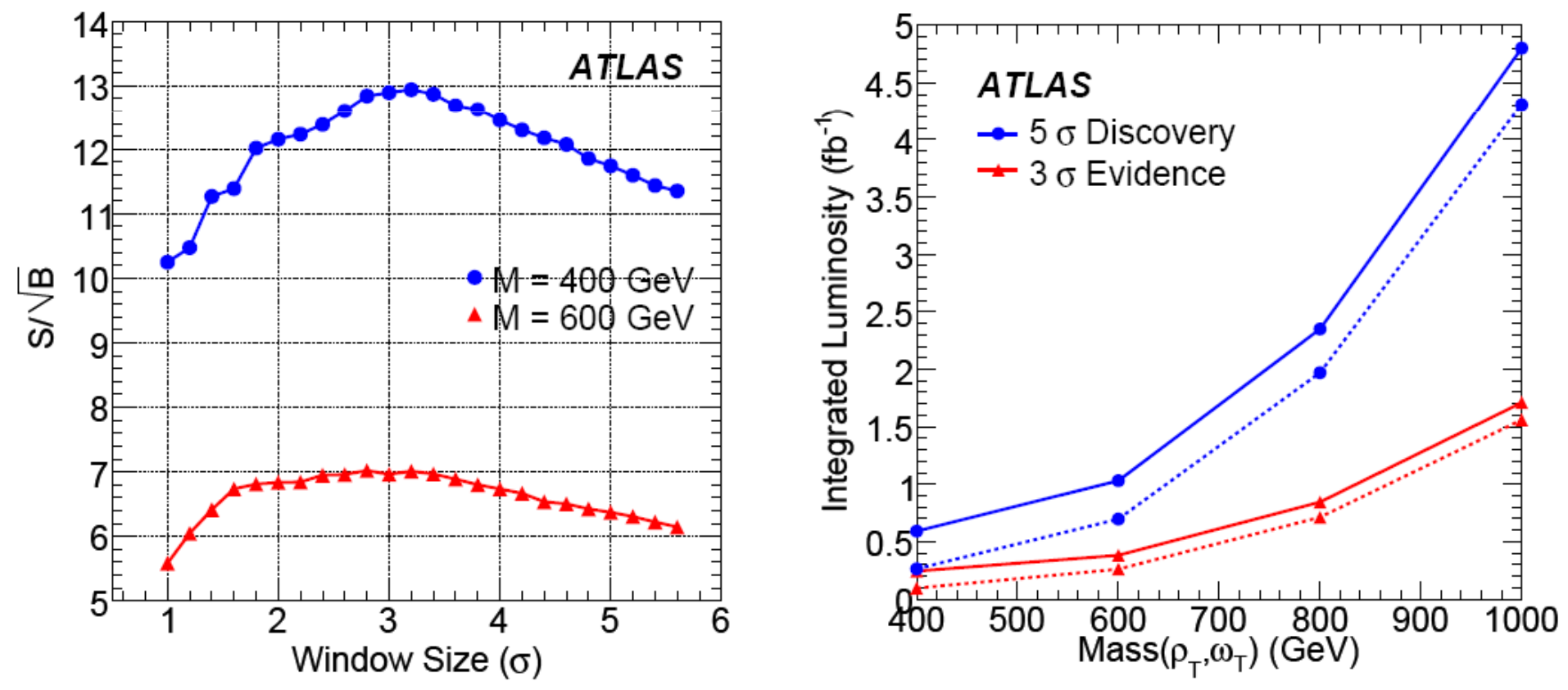

- Including estimated early alignment: $+50 \%$ luminosity needed 


\section{DILEPTONS}

$\tau \tau$ 
- Tau leptons can decay hadronically or leptonically

- All modes (hh, Ih, II) have been studied and combined in the search

- Selection: Missing energy, upper bound on transverse mass and $\mathrm{p}_{T}{ }^{\text {TOT }}$, b-jet veto.

- Tevatron limits: $400 \mathrm{GeV}$

- Neutrinos are always present

Still, the collinear approximation allows the reconstruction of the invariant mass

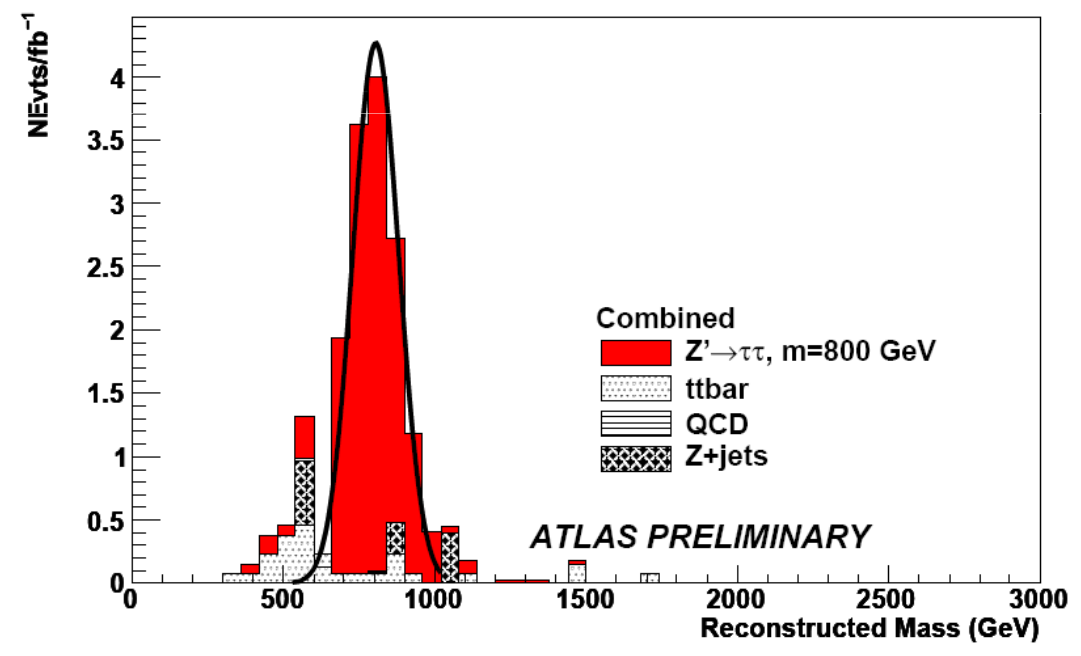

... except when the tau leptons are back-to-back $\rightarrow$ "visible mass" $\mathrm{m}_{\mathrm{vis}}$

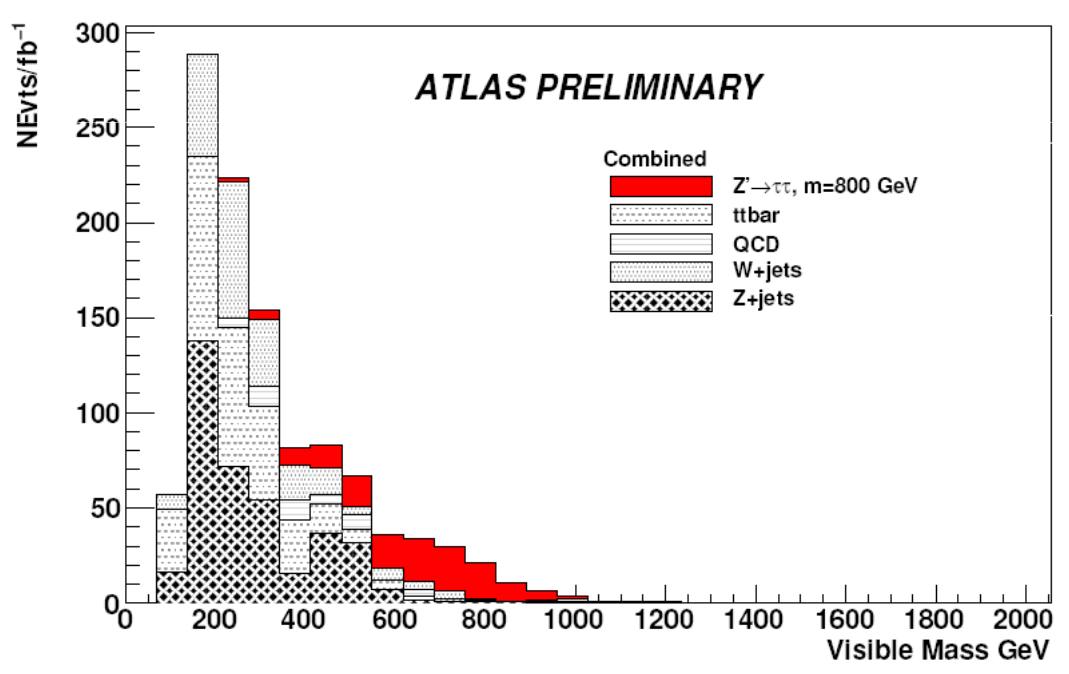

- In the collinear approximation, the not-back-to-back requirement, plus cuts on solutions that are physical, reduce both signal and backgrounds 


\section{Sensitivity}

- $\mathrm{Z}^{\prime}{ }_{\text {SSM }}$ with a mass up to 1.2 TeV could yield a 5 sigma significance with $\sim 1 \mathrm{fb}^{-1}$ of data
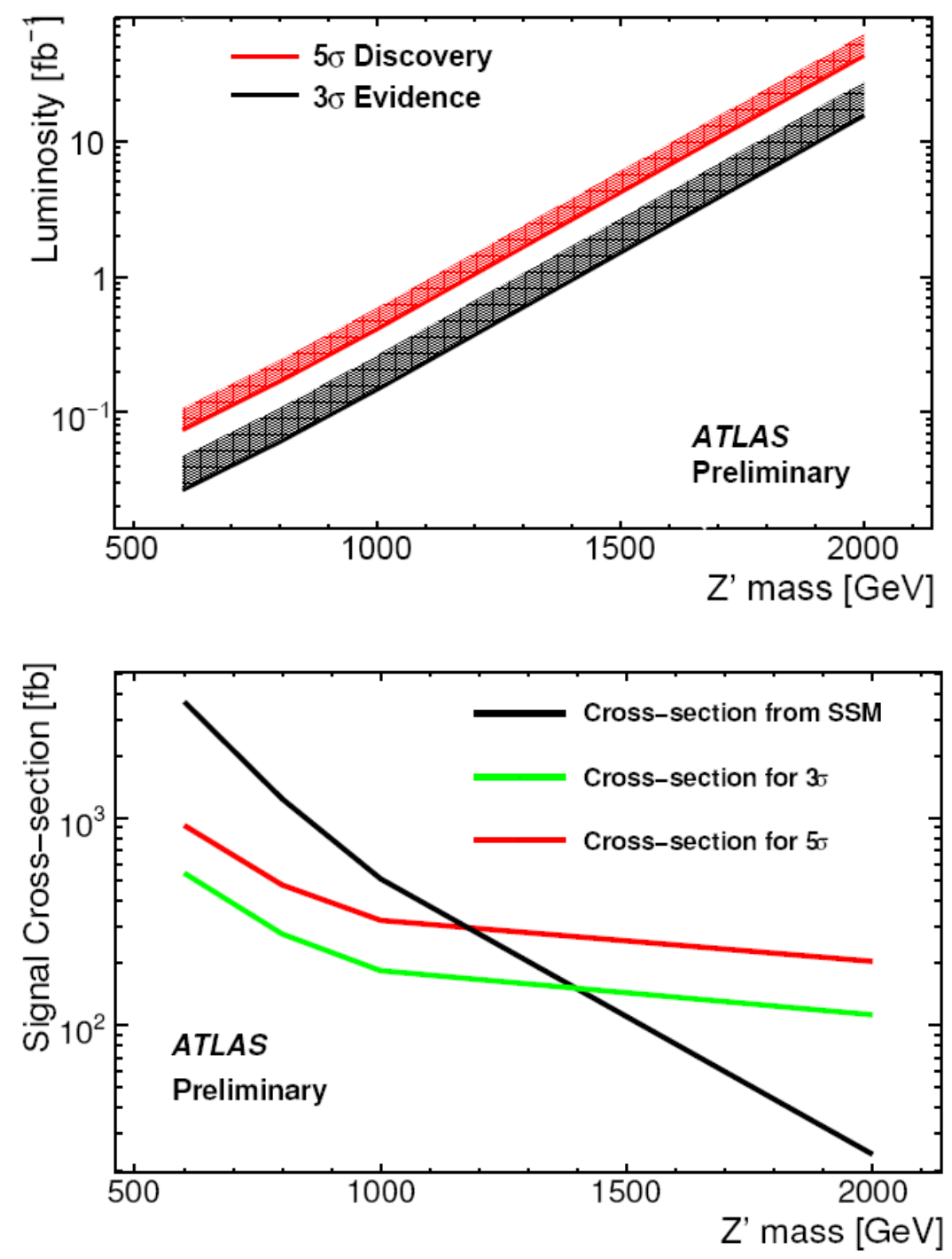


\section{LEPTON - NEUTRINO}




\section{Signature, selection}

- Several BSM scenarios include heavy, narrow, charged gauge bosons able to decay into I+V

- As with SM W, the transverse mass helps extract them

$$
m_{T}=\sqrt{2 p_{T} \mathscr{E}_{T}\left(1-\cos \Delta \phi_{\ell, E_{T}}\right)}
$$

- Rejecting events with high jet activity, the main remaining background is the high tail of the SM W boson.

- Mis-reconstructed leptons (low Pt reconstructed as high $\mathrm{Pt}$ ) are a concern for early data

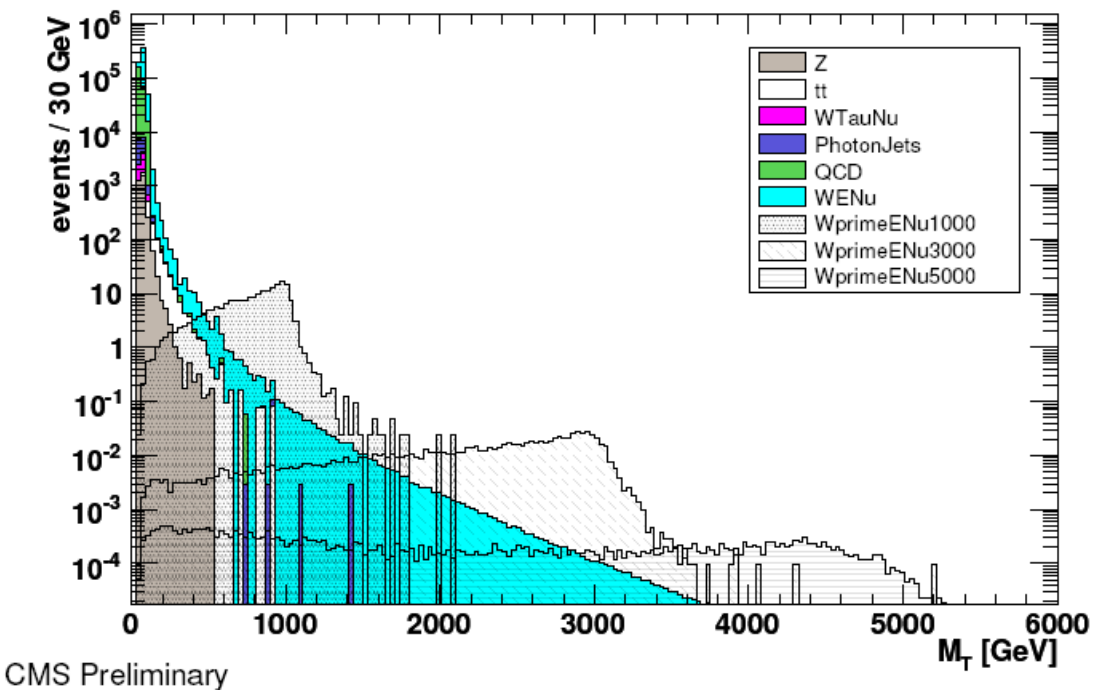




\section{Selection}

- One high- $\mathrm{p}_{\mathrm{T}}$ lepton

- Missing energy

- Lepton fraction (ATLAS), $\mathrm{E}_{\mathrm{T}} / \mathrm{MET}$ (CMS)

- Jet Veto
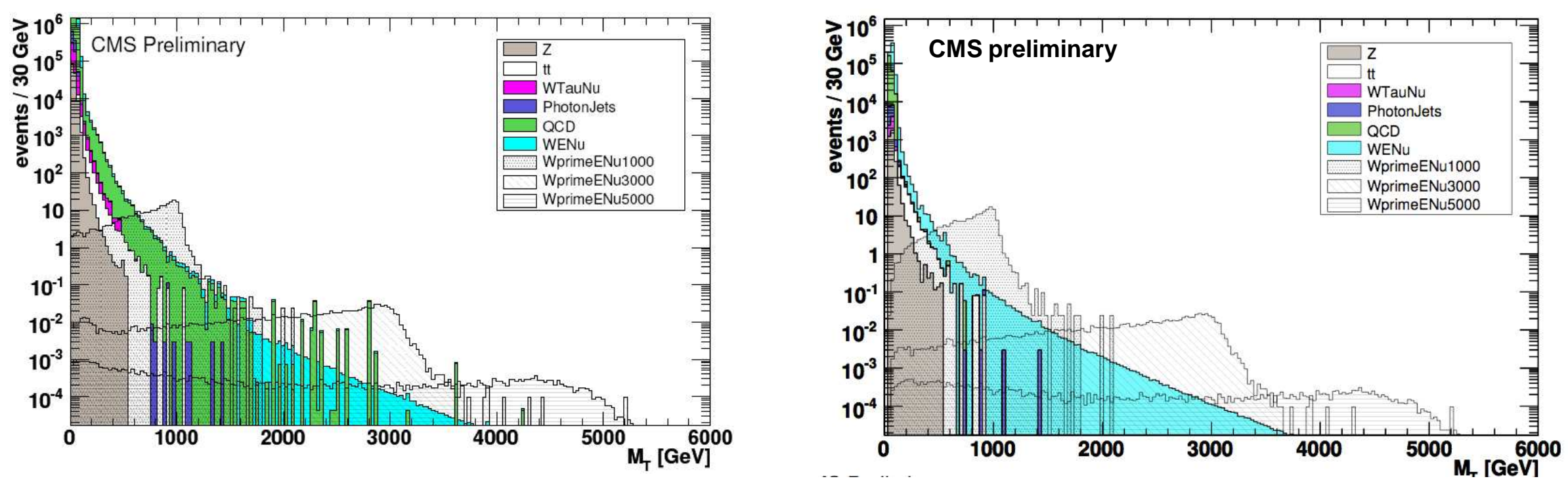


\section{Electron, muon channels}

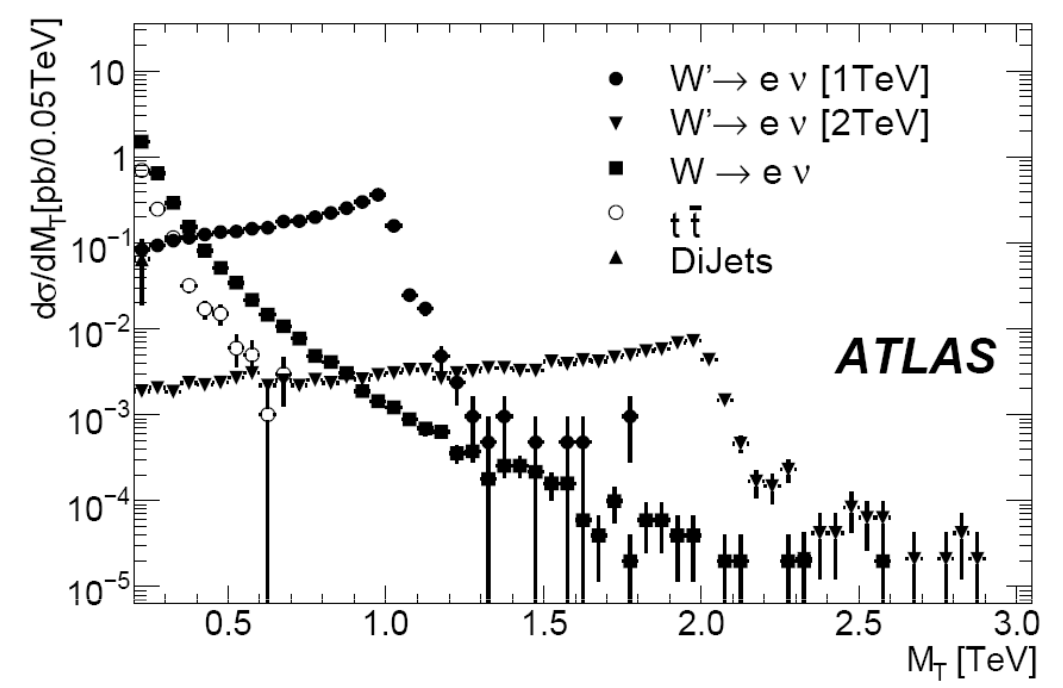

- Electron, muon channels studied

- Worse muon resolution at high $\mathrm{p}_{\mathrm{T}}$

- Possible discovery above TeV limits (1TeV) with $\mathrm{O}\left(10 \mathrm{pb}^{-1}\right)$
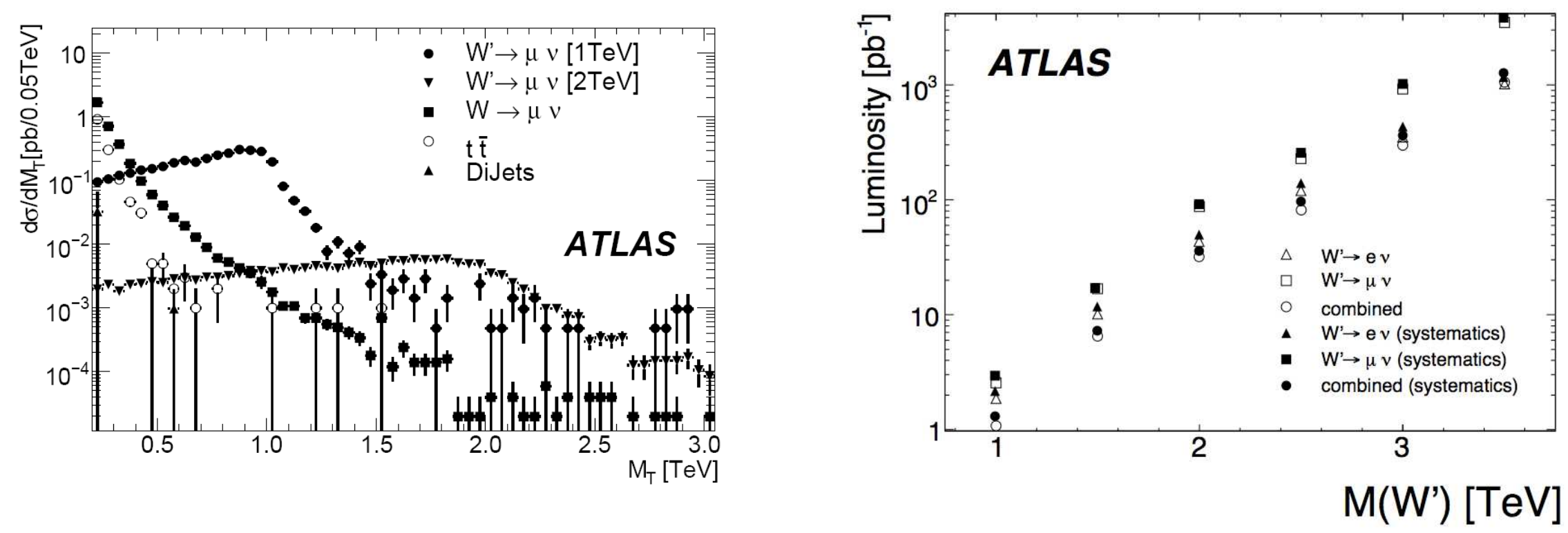


\section{Discovery potential}
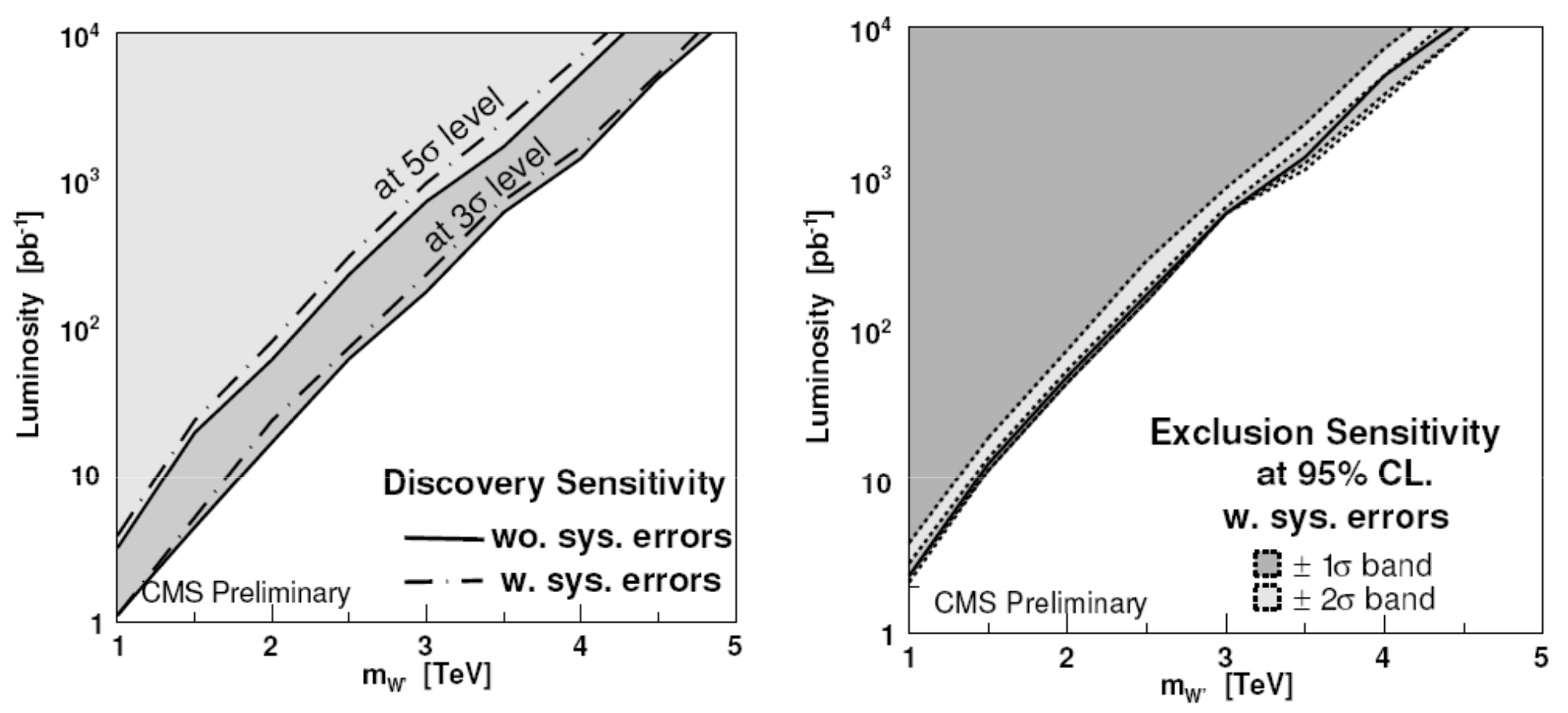

- With $\mathrm{O}\left(200 \mathrm{pb}^{-1}\right)$, masses up to $\sim 2.5 \mathrm{TeV}$ can be probed

- Limits up to 3TeV 


\section{LEPTONS + JETS}




\section{Leptons+jets?}

- Leptoquarks

- Bosons carrying quark and lepton number

- Experimental constraints favor three generations, each coupling to a SM generation

- mLQ1 > $256 \mathrm{GeV}(\mathrm{D} \varnothing)$

- mLQ2 > $251 \mathrm{GeV}(\mathrm{D} \varnothing)$

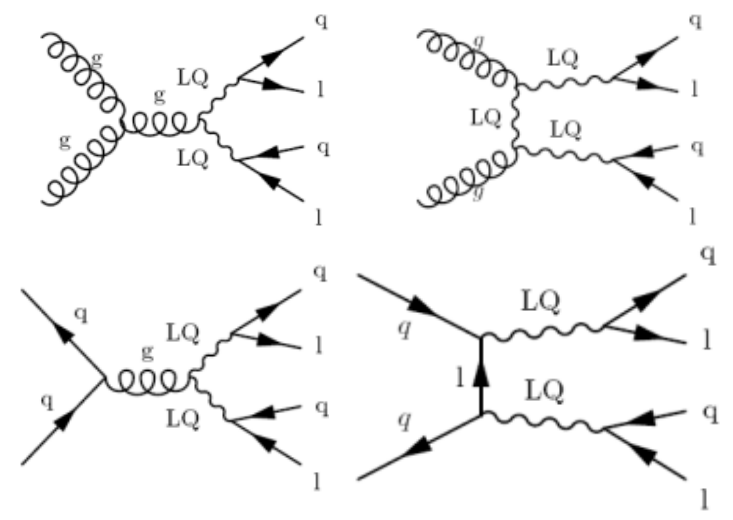

- Left-Right Symmetric Models (LRSM)

- Address non-zero neutrino mass and baryogenesis

- Three heavy right-handed Majorana neutrinos $\left(\mathrm{N}_{\mathrm{e}}, \mathrm{N}_{\mu}, \mathrm{N}_{\tau}\right)$

- Some LRSMs introduce $W_{R}$ and $Z^{\prime}$

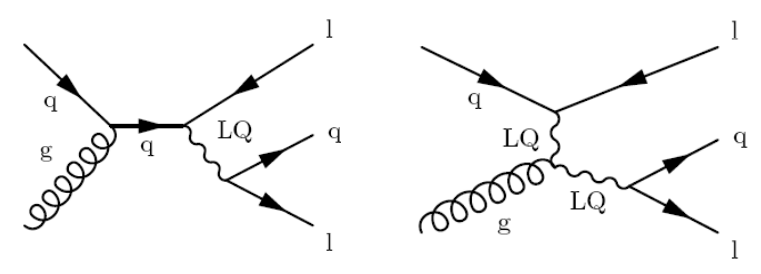




\section{Leptoquarks}

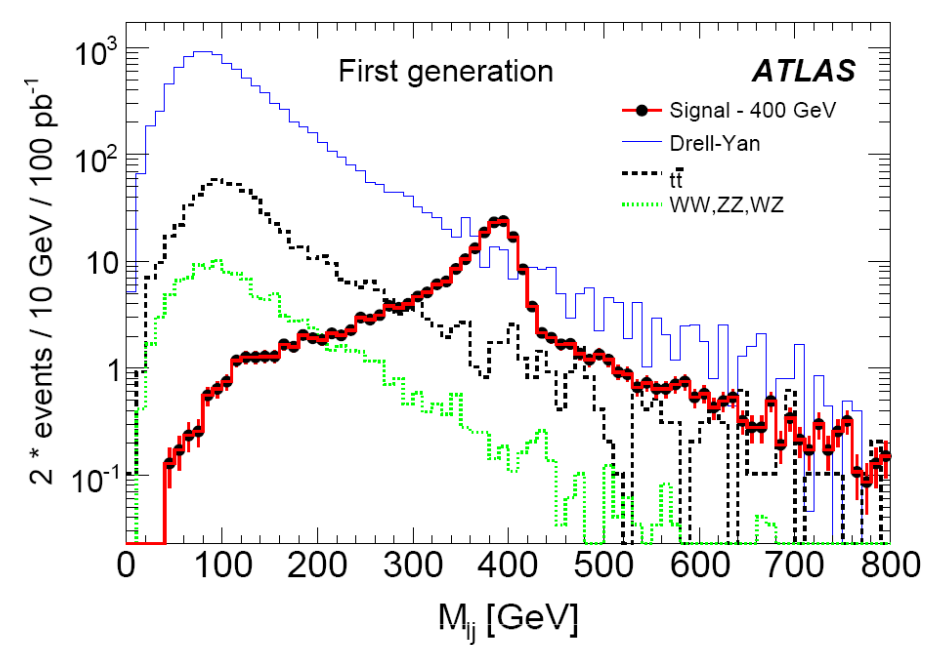

- 2 leptons (opp charge, same flavor)

- At least 2 jets

- Background rejection:

- Leptons transverse momenta

- $\quad \mathrm{S}_{T}=\sum\left|\vec{p}_{T}\right|_{\text {jet }}+\sum\left|\vec{p}_{T}\right|_{\text {lep }}$

- Dilepton invariant mass

- Lepton-jet invariant mass
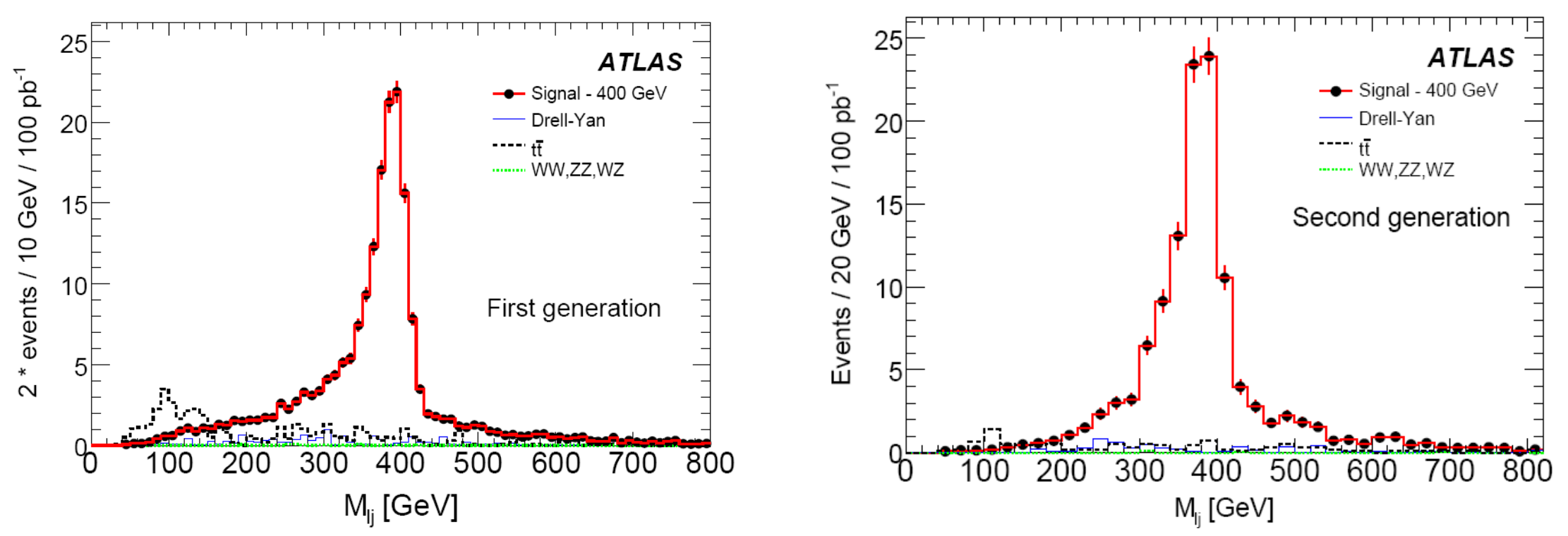


\section{LRSM}

- Majorana Neutrino masses

- $\mathrm{W}_{\mathrm{R}}$
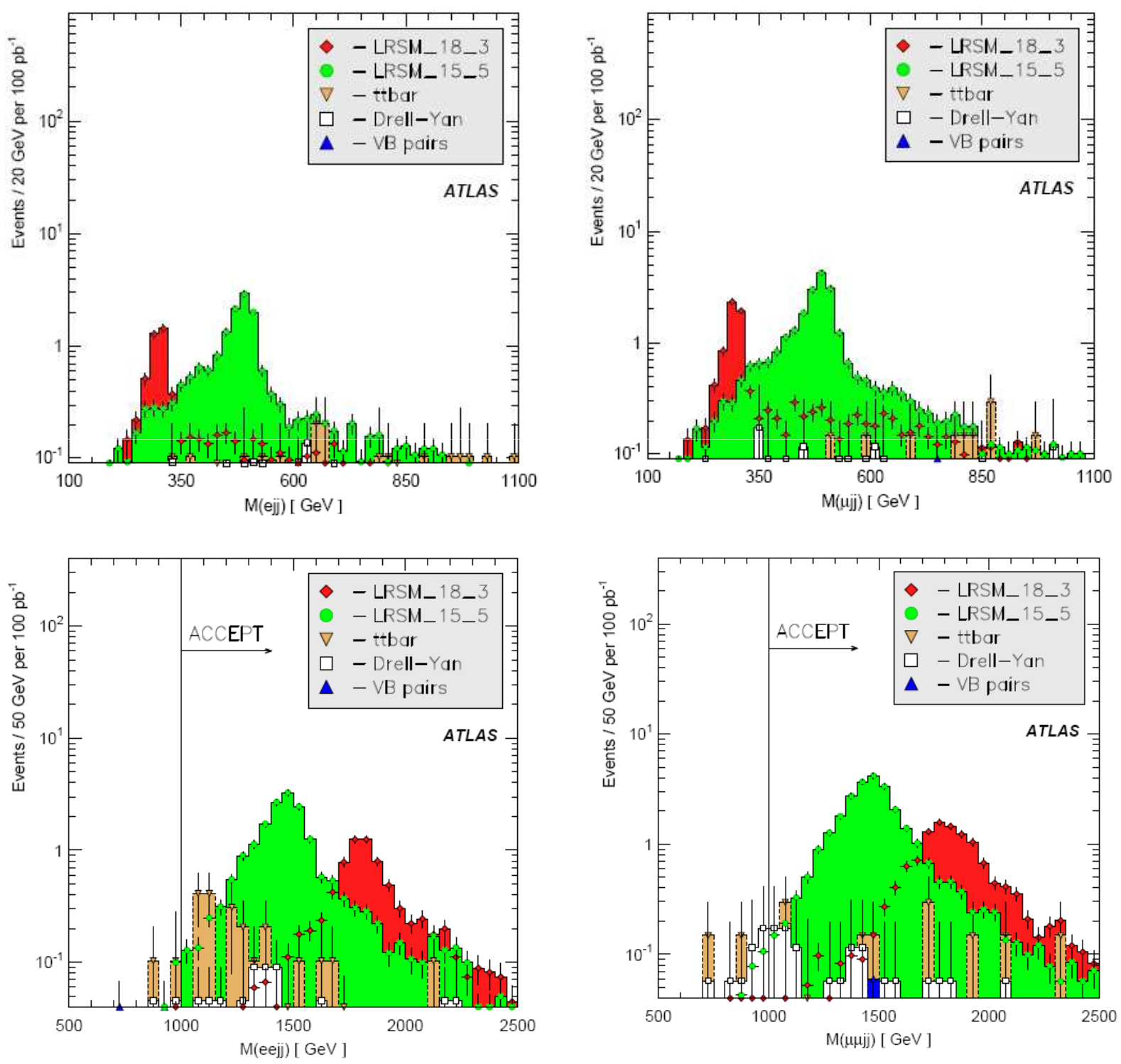


\section{Sensitivity}
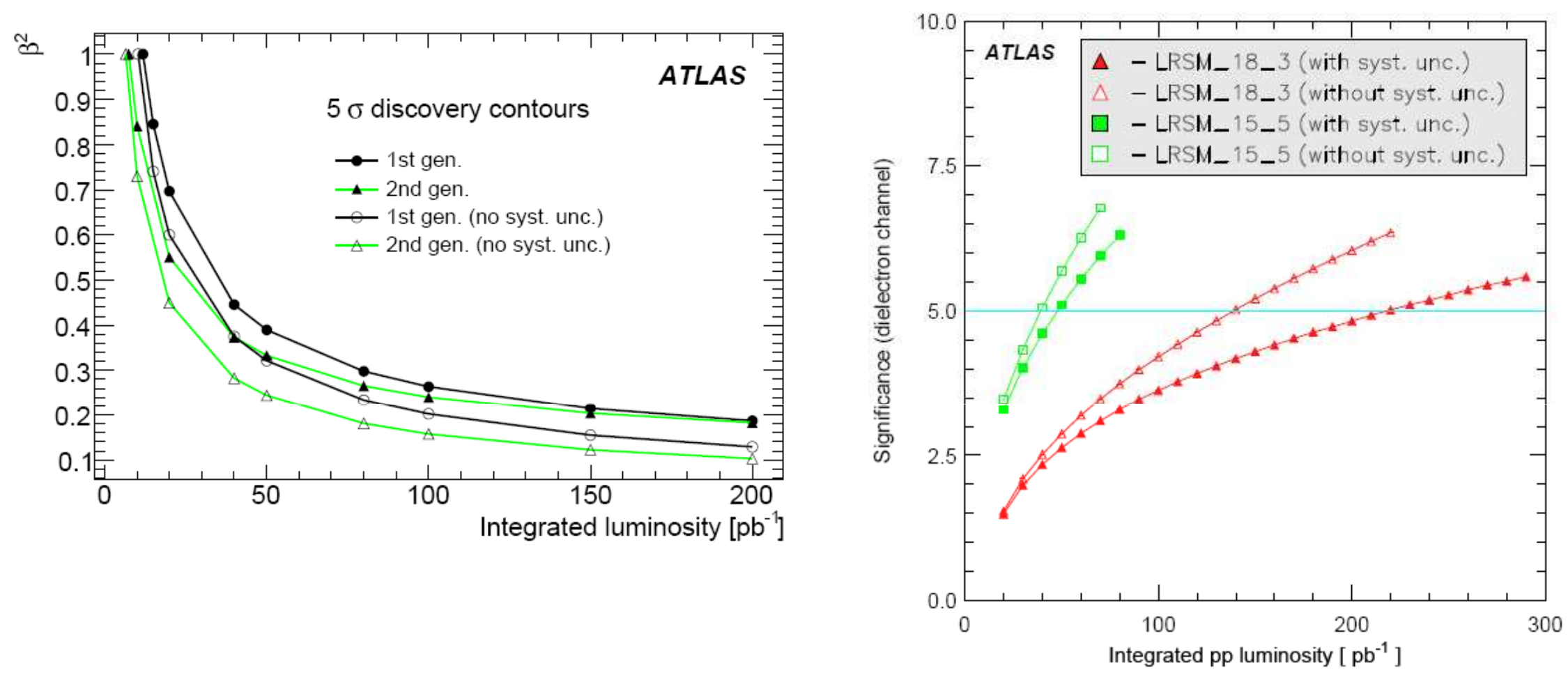

- Both types of models could yield a 5sigma signal with $\mathrm{O}(100 \mathrm{pb}-1)$ 


\section{CONCLUSIONS}


- Several plausible extensions of the SM predict narrow resonances

- Background estimation procedures, fit-based strategies, statistical tools have been developed

- $14 \mathrm{TeV}$ studies have shown that they could be established at the 5 sigma level even with $\mathrm{O}(100 / \mathrm{pb})$ of integrated luminosity

- The lowered center-of-mass energy, at 10TeV, degrades the sensitivity, but the initial run $(\mathrm{O}(200 / \mathrm{pb}))$ should still be enough to go beyond Tevatron limits in most of these models

- Looking forward to the start of collision data! 


\section{References}

- CERN-OPEN-2008-020 (ATLAS, arXiv:0901.0512)

- CERN-LHCC-2006-021 (CMS, J.Phys.G: Nucl.Part.Phys.34 995-1579)

- https://twiki.cern.ch/twiki/bin/view/CMS/PhysicsResults 


\section{BACKUP}




\section{Electrons}

- The QCD cross sections at LHC are 10 to 100 times

- higher than at the Tevatron :

- @Pt $=40 \mathrm{GeV} / \mathrm{c}$ : electron to jet ratio is $~ 10-5$
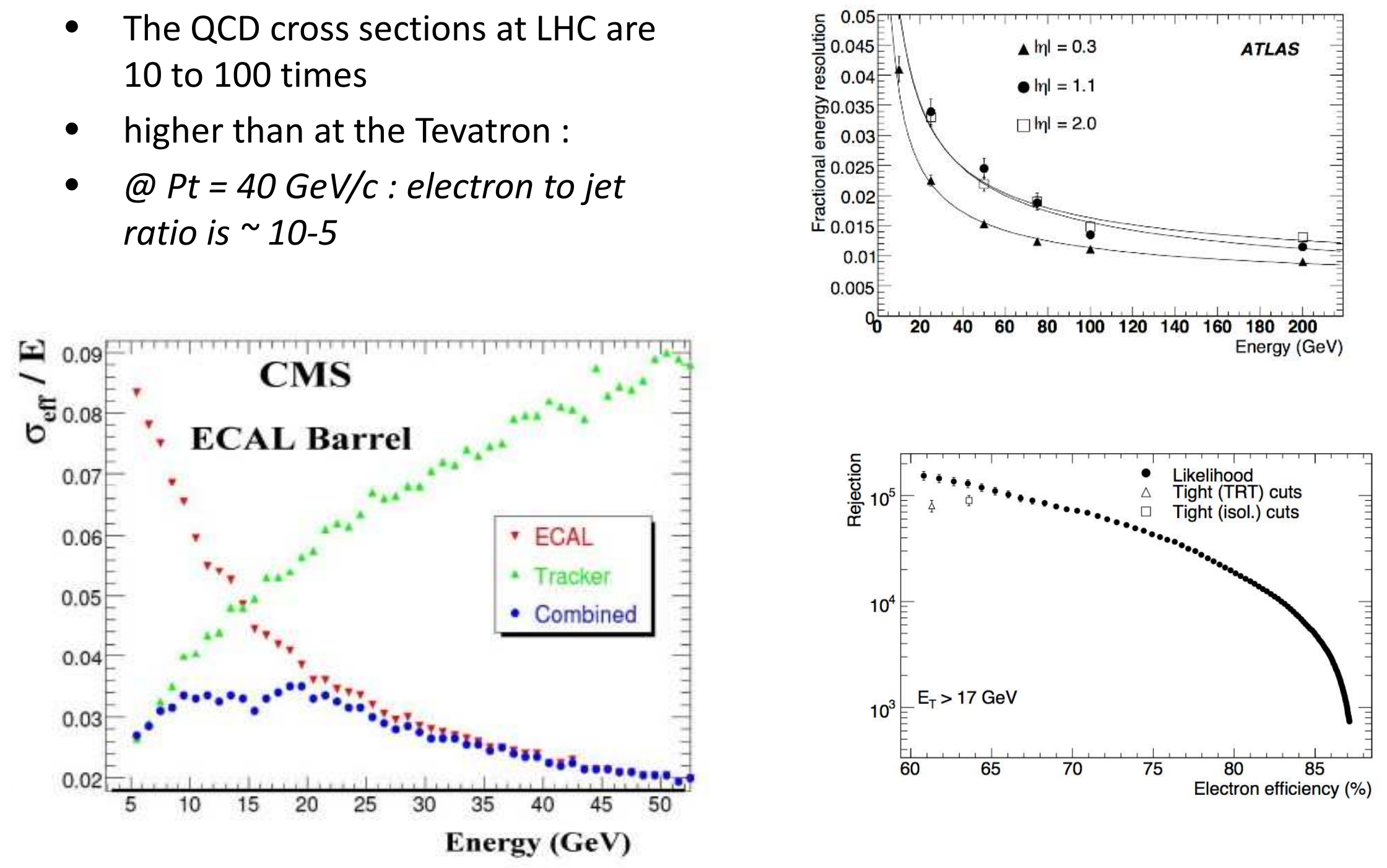


\section{Muons}
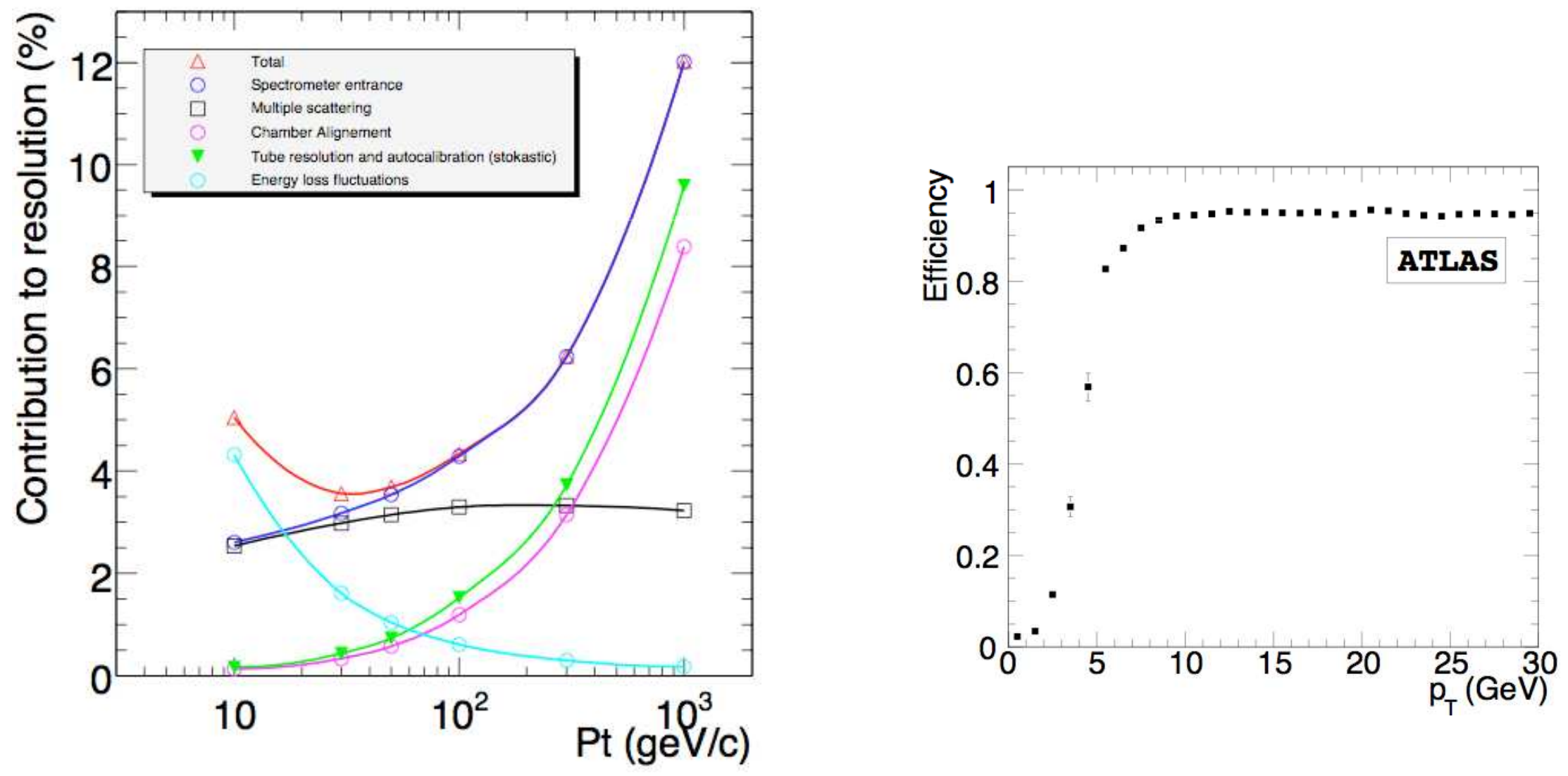


\section{Tau leptons}

- With 100 pb-1, clear signals for $\mathrm{W}$ and $\mathrm{Z}$ in $\tau$ channels

- $Z \rightarrow \tau \tau$ can then be used to set the ET miss scale to a few $\%$

- $\tau$ reconstruction is tricky and relies (not for very first data but soon after) on multivariate techniques.
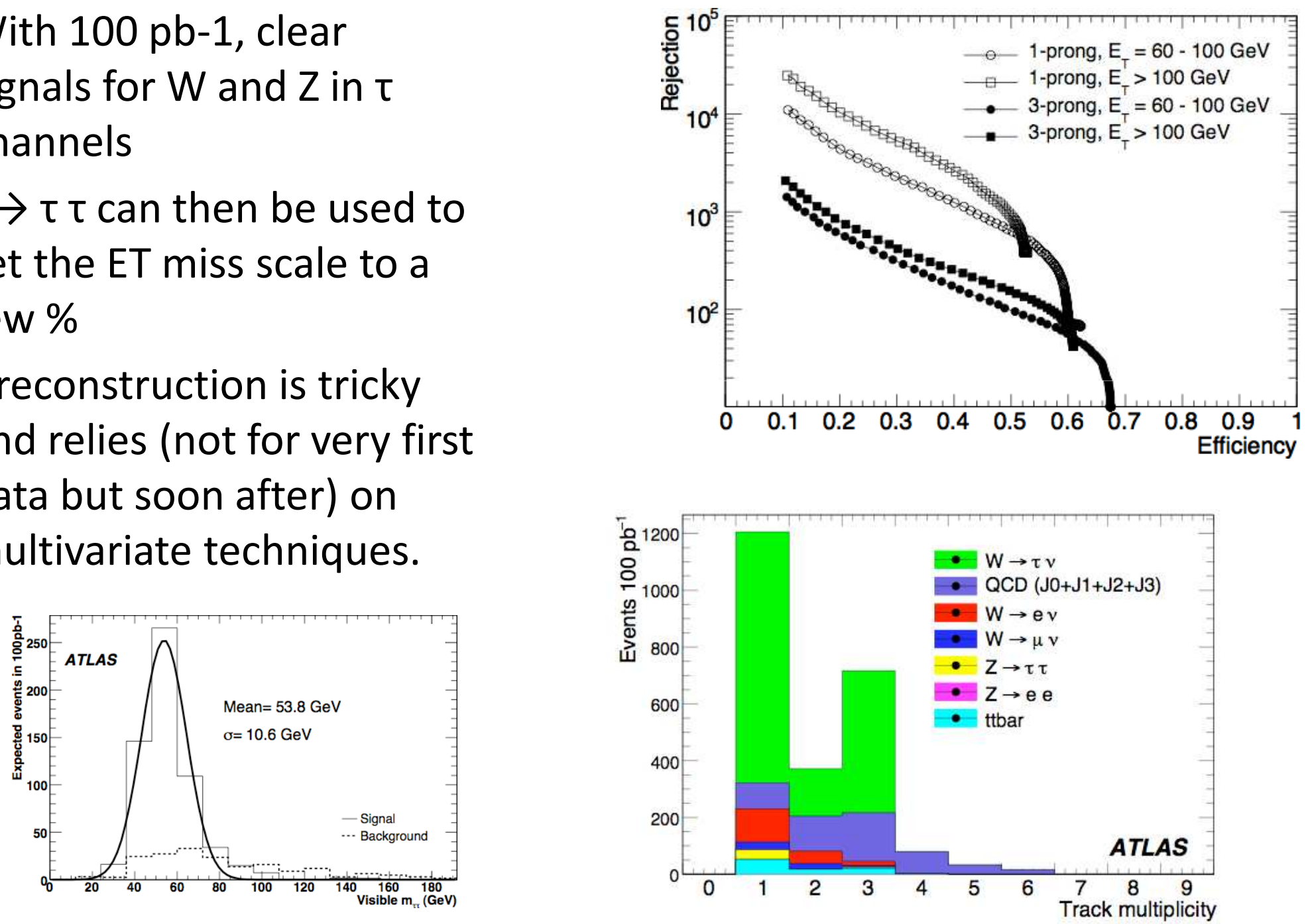